\title{
ROTTE ANGIOINE. TRASMISSIONE DI MODELLI FIGURATIVI E MOBILITÀ DEGLI ARTISTI TRA NAPOLI E IL VERSANTE ADRIATICO DEL REGNO: IL CASO DI SAN DOMENICO A PENNE
}

\section{PAOLO DI SIMONE}

UDC: $726.54(450.655)$ $75.052 " 13 "$

Review

Manuscript received: 14. 12. 2015.

Revised manuscript accepted: 09. 03. 2016.

DOI: 10.1484/J.HAM.5.111352
P. di Simone

Dipartimento di Lettere, Arti e Scienze Sociali Facoltà di Lettere e Filosofia Università di Chieti "G. d'Annunzio" via dei Vestini, 31, 6610o Chieti, Italia paolodisimone@tiscali.it

This essay takes into account the fragments of a mid-fourteenth-century fresco cycle in the church of San Domenico in Penne, representing biblical stories, the legend of Saint John the Evangelist and, probably, the visions of Apocalypse. These frescoes reveal stylistic parallels with contemporary Neapolitan miniature and painting, and seem to attest the mobility of artists and the transfer of form between Naples, with its giottesque masterpieces in the church of Santa Chiara and in the Palatine Chapel in Castel Nuovo, and the Adriatic side of the angevin Kingdom.

Keywords: Penne, Naples, Giotto, Angevins

1. Chi entra oggi nella chiesa di San Domenico a Penne (fig. 1), difficilmente si accorgerà di trovarsi all'interno di un edificio medievale: l'aula è rivestita di stucchi e altari barocchi, che si susseguono sinuosi fino al coro, occultato da una mensa scenografica; la luce penetra da alte finestre, quasi nascoste da una movimentata modanatura sulla quale poggiano gli archi delle volte e i pennacchi della cupola; i colori dominanti sono il grigio e l'ocra, interrotti da enormi cornici che accolgono dipinti realizzati tra il XVI e il XVIII secolo. La chiesa fu infatti trasformata radicalmente tra il 1722 e il 1730 per opera del ticinese Giovanni Battista Gianni, autore del progetto, e di Girolamo Rizza, la cui bottega di plasticatori si accinse a decorare l'interno, aggiornandolo ai nuovi canoni estetici'.

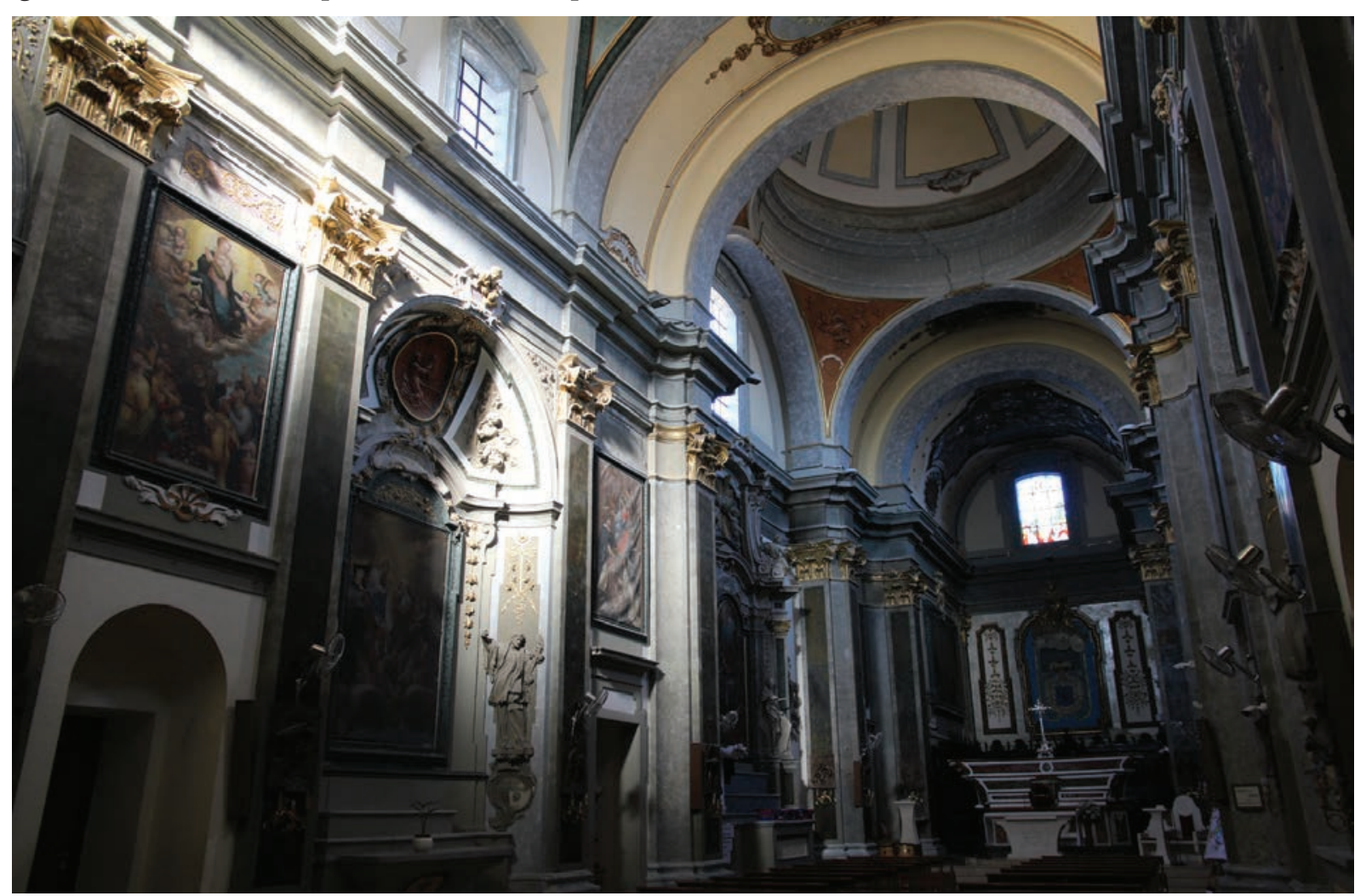

Fig. 1 - Penne, Chiesa di San Domenico, interno

${ }^{1}$ F. BATTISTELLA, Note su alcune fabbriche attribuite a Francesco di Sio architetto napoletano attivo in Abruzzo tra il settimo e il nono decennio del XVIII secolo, in Rivista Abruzzese. Rassegna trimestrale di cultura, 42, 1989, 2, pp. 97-183: pp. 130-132 nota 46 e 139-145 nota 65 (in part. pp. 142-143); ID., La decorazione a stucco della chiesa di San Giovanni Battista di Penne e altre opere di stuccatori intelvesi in territorio vestino, in Dalla valle del Fino alla valle del medio e alto Pescara, Pescara, 2003 (Documenti dell'Abruzzo Teramano, VI/1), pp. 580-593: 588; P. DI SIMONE, Penne. Il Barocco scomparso, in R. Torlontano (ed.), Abruzzo. Il Barocco negato. Aspetti dell'arte del Seicento e Settecento, Roma, 2010 (Atlante tematico del Barocco in Italia), pp. 283-291: 283. 


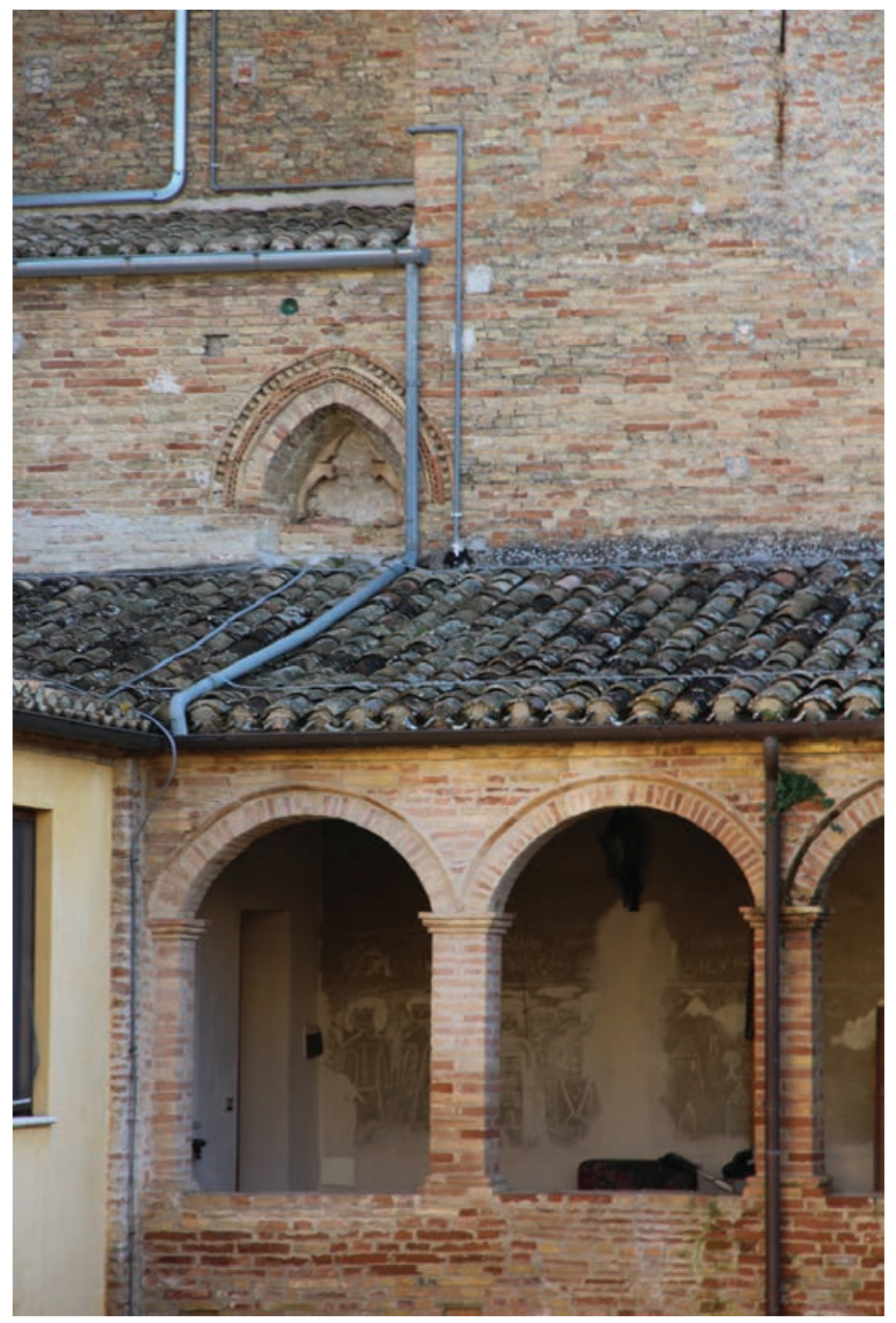

Fig. 2 - Penne, Chiesa di San Domenico, fianco meridionale, monofora

Per imbattersi in testimonianze più antiche è necessario uscire nel chiostro, dov'è ancora in parte visibile l'originario paramento in laterizio del fianco settentrionale, ritmato da strette monofore lanceolate, chiuse in epoca moderna (fig. 2) $)^{2}$.
Quasi tutta perduta, purtroppo, è anche la documentazione d'archivio relativa al periodo medievale3: lasciando da parte i ricordi dei frati pennesi Frugerio, presente il 17 agosto 1233 al processo di canonizzazione di Domenico di Guzmán ${ }^{4}$, e Giovanni, priore di Santa Sabina a Roma nel $1235^{5}$, il primo indizio certo della presenza domenicana in città rimonta al 1283, anno in cui il convento risulta funzionante assieme a quelli dell'Aquila e di Sulmona ${ }^{6}$. Di grande importanza è un atto del 20 novembre 1294, con cui Carlo II d'Angiò istituisce una rendita settimanale di un fiorino a favore di una serie di cenobi domenicani sparsi nel territorio del Regno: tra i beneficiari, il nostro spicca al fianco di quello napoletano di San Pietro Martire eai conventi di Benevento, Gaeta, Sessa, Capua, Aversa, Somma, Salerno, Foggia, Manfredonia, Trani, Bari, Monopoli, Brindisi, Venosa, Sulmona, Chieti, Atri e Ortona ${ }^{7}$. L'eco di questo antico legame con la corte angioina sopravvive nelle fonti moderne, che riportano la tradizione secondo la quale il cenobio fu fondato per volontà dello stesso sovrano ${ }^{8}$. Ad altre testimonianze trecentesche accenneremo più avanti.

Le più significative vestigia medievali si trovano tuttavia all'interno della chiesa, seppure occultate agli occhi del visitatore dalla moderna parete di testata del coro: ci riferiamo ai resti di un grande ciclo pittorico trecentesco, custoditi in un ambiente situato sopra l'attuale sagrestia, raggiungibile tramite una scala che conduce al campanile e al secondo ordine del chiostro. L'aula fu infatti ridotta in lunghezza in tempi non precisabili, e l'antica zona absidale tagliata longitudinalmente in due da un solaio. Nell'angusto sottotetto, dimenticati, i lacerti hanno resistito alle successive modifiche, fino alla loro riscoperta e valorizzazione negli anni Novanta del XX secolo.

Già noti alla letteratura locale 9 , gli affreschi sono stati fatti oggetto di una breve ma acuta analisi da Pierluigi Leone de Castris $^{10}$, che ha correttamente decifrate molte delle scene superstiti e ne ha proposta una collocazione stilistica nell'àmbito della bottega del cosiddetto Maestro di Offida ${ }^{11}$, attiva soprattutto nel Piceno tra il quarto e il settimo decennio del Trecento e caratterizzata dapprima dalla conoscenza di stilemi marchigiano-riminesi e poi dall'improvviso accoglimento di elementi giotteschi napoletani ${ }^{12}$ : secondo l'ipotesi dello studioso, a episodi dell'Infanzia di Cristo (sulla parete

\footnotetext{
${ }^{2}$ Simili monofore si aprivano anche sulla parete meridionale, oggi occultata da edifici novecenteschi. Sono tuttavia visibili in una fotografia successiva alla Seconda Guerra Mondiale, in collezione privata, raffigurante il Teatro cittadino, in origine addossato alla chiesa, ridotto in macerie dai bombardamenti.

${ }^{3}$ Le lacune documentarie erano già lamentate nel 1756, quando si scriveva che «circa la sua Fondazione [scil. della chiesa] non si ha niente di certo, a cagione, che gl'Archivi (come si ha per tradizione) per le guerre furono abbrugiati»: Roma, Archivio Generalizio dell'Ordine dei Predicatori (d'ora in avanti AGOP), XIV, Litt. A, f. 252, cit. in B. CARDERI, I Domenicani nella diocesi di Penne, in Bullettino della Deputazione Abruzzese di Storia Patria, 64 (96), 1974, 2, pp. 667-953: 861 .

${ }^{4}$ B. CARDERI, I Domenicani nella diocesi di Penne (cit. nella nota precedente), pp. 669-670 e 734-736.

${ }^{5}$ Ibidem, pp. 671, 736.

${ }^{6}$ Ibidem, pp. 670-671 e 736-737.

${ }^{7}$ AGOP, XIV, Litt. A, ff. 468v-689r, 20 novembre 1294, cit. in Ibidem, p. 737: «Re Carlo II d'Angiò dona per qualsivoglia settimana all'infrascritti conventi dell'Ordine de' Predicatori, cioè uno fiorino d'oro a Napoli (S. Pietro Martire), Benevento, Gaeta, Sessa, Capua, Aversa, Somma, Salerno, Foggia, Manfredonia, Trani, Bari, Monopoli, Brindisi, Venosa, Sulmona, Penne, Chieti, Andria (Atri), Ortona; tre fiorini a S. Domenico Maggiore di Napoli; due fiorini d'oro ai conventi di Aquila e Barletta; comandando di pagarsi in due termini, cioè la metà nella festa del Natale del Signore, e l'altra metà nella festa della Resurrezione».

${ }^{8}$ AGOP, XIV, Litt. A, f. 252r, cit. in Ibidem, p. 861: «comunemente (...) si tiene che fu uno de 12 Conventi fondati dal Re Carlo d'Angiò in questo regno di Napoli». 9 C. GRECO, Città di Penne. Beni artistici e architettonici, Penne, 1999, p. 25; A. PROCACCI, M. COSTANTINI, Note storiche di Penne, Penne, 2000 , pp. 21-22. Un dettaglio degli affreschi è altresì riprodotto in S. DELL'ORSO, Un itinerario attraverso gli affreschi abruzzesi del primo Quattrocento (Calendario Tercas 1992), tav. XII fig. 10.

${ }^{10}$ P. LEONE DE CASTRIS, Gli affreschi dell'antico coro della chiesa di San Domenico e l'attività del presunto Luca d'Atri a Penne, in Dalla valle del Fino alla valle del medio e alto Pescara, op. cit. (n. 1), pp. 480-482.

${ }^{n}$ La ricostruzione dell'attività di questo maestro è avviata in F. BOLOGNA, Santa Maria ad Ronzanum, in La valle Siciliana o del Mavone, Roma, 1983 (Documenti dell'Abruzzo Teramano, I/1), pp. 147-234: 224-225 e, soprattutto, nel memorabile F. BOLOGNA, P. LEONE DE CASTRIS, Percorso del Maestro d'Offida, in Studi di storia dell'arte in onore di Mario Rotili, Napoli, 1984, pp. 283-305. Per quanto riguarda le successive conclusioni di Leone de Castris si
} 


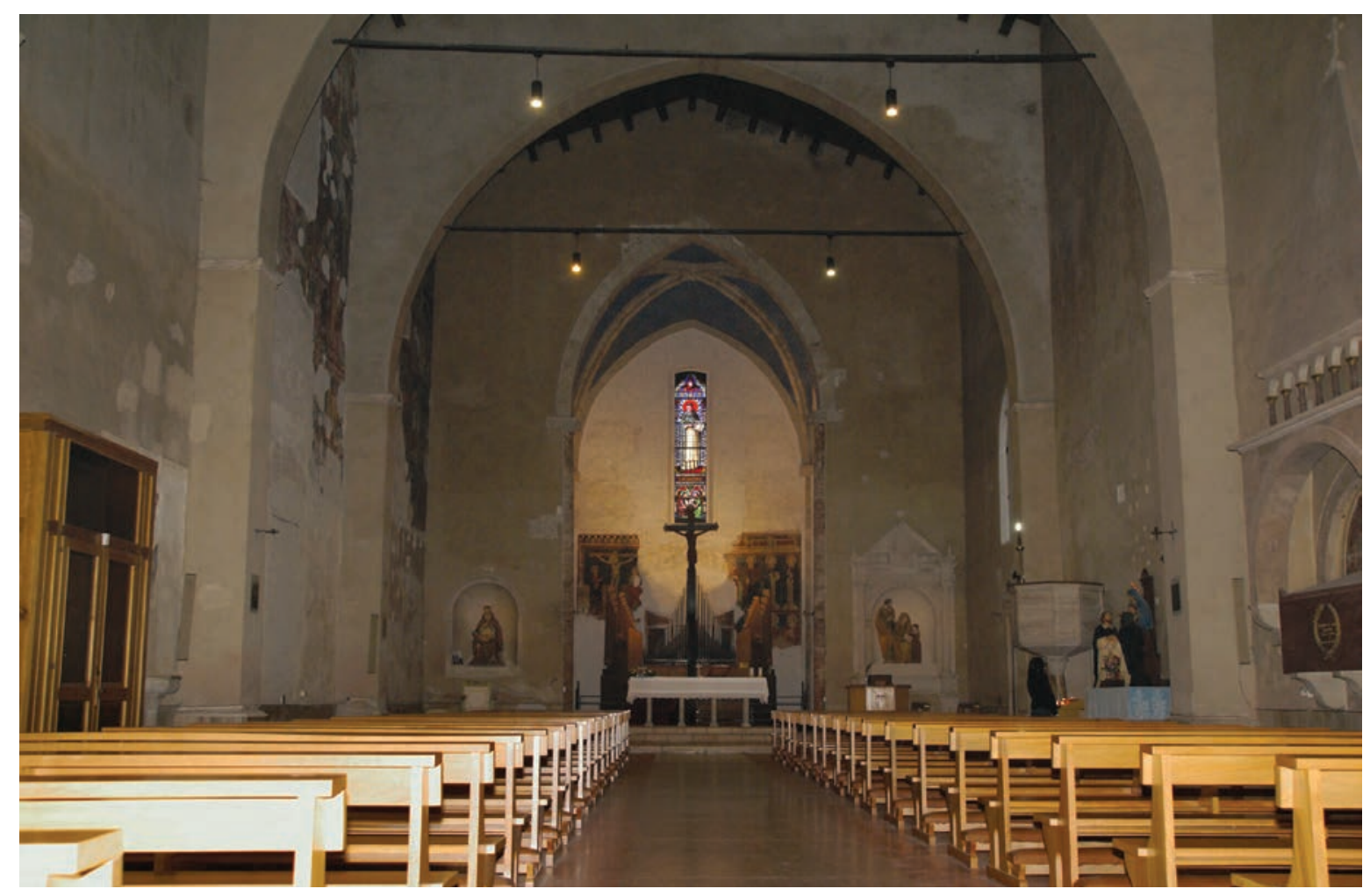

Fig. 3 - Teramo, Chiesa di San Domenico, interno

sinistra: la Strage degli Innocenti, la Presentazione al Tempio e, forse, una perduta Adorazione dei Magi o dei pastori nel soprastante lunettone; su quella di fondo, Gesù tra i Dottori) dovevano accompagnarsi delle scene di martirio ${ }^{13}$, magari di uno dei santi protettori della città ${ }^{14}$ (nella parete di testata, probabilmente, una preparazione al supplizio; in quella di destra, il sacrificio di un santo giovane, «in preghiera e in apparenza cavaliere, salvato dall'intervento divino dalle fiamme di un rogo») $)^{15}$. Senza addentrarci per ora nel dibattito relativo all'analisi formale, vediamo se è possibile precisare meglio la lettura iconografica delle superstiti figurazioni.
2. Il grande ciclo occupava tutta la superficie muraria dell'abside e, come vedremo, dello stesso arco absidale ${ }^{16}$. L'interno trecentesco della chiesa doveva infatti essere identico a quello, ad esempio, del San Domenico di Teramo (fig. 3) ${ }^{17}$ : l'aula unica, coperta a capriate lignee, culminava in un'abside a pianta rettangolare con volta a crociera, illuminata da una alta e stretta monofora che si apriva sulla parete di fondo. L'icnografia è senz'altro quella tipica di molte fondazioni mendicanti (senza spostarci da Penne, è la stessa della chiesa degli Eremitani, prima dei consueti rifacimenti barocchi) ${ }^{18}$, ma anche di edifici simbolo della Napoli angioina come la

vedano, con bibliografia pregressa, P. LEONE DE CASTRIS, Appendice. Pittura del Trecento nell'Abruzzo Teramano, in Teramo e la valle del Tordino, Teramo, 2006 (Documenti dell'Abruzzo Teramano, VII/1), pp. 440-453: 442-445, e ID., Il Maestro di Offida nell'Abruzzo teramano, in Civiltà urbana e committenze artistiche al tempo del Maestro di Offida (secoli XIV-XV), atti del convegno (Ascoli Piceno, 2011), a cura di S. Maddalo e I. Lori Sanfilippo, Roma, 2013, pp. 103-115 (nello stesso volume, si veda anche F. BOLOGNA, Il Maestro di Offida, alle pp. 321-326). Tra gli altri studi relativi al Maestro, segnaliamo senz'altro S. PAPETTI, Proposta per il Maestro di Offida ed i suoi seguaci ad Ascoli Piceno, in Notizie da Palazzo Albani, 16, 1988, 1, pp. 139-148; A. TARTUFERI, Qualche osservazione sul Maestro di Offida e alcuni appunti sulla pittura del Trecento nell'Abruzzo teramano, in Arte Cristiana, 88, 2000, 799, pp. 249-258; S. PAPETTI, Pittura, in Atlante del gotico nelle Marche. Ascoli Piceno e provincia, Milano, 2004, pp. 42-72: 42-57, 68-71; E. ZAPPASODI, Consonanze pittoriche trecentesche di qua e di là dell'Appennino, in Spoletium, 4, 2011, pp. 32-43 e M. D'ATTANASIO, Il Maestro d'Offida ad Atri. Lo status qurestionis, in A. Madonna, M.C. Rossi (ed.), Atri e la sua cattedrale prima degli Acquaviva, atti del convegno (Atri, 2013), Pescara, 2015, pp. 90-101, con ulteriori additamenti bibliografici.

${ }^{12}$ Come giustamente rilevato dallo studioso, bene evidenti in questo ciclo, nel quale «poco si coglie [...] dell'originaria formazione marchigiano-riminese del pittore: le architetture sono più evolute e di marca più spiccatamente giottesca e masiana [...]; ed anche le figure, insistentemente - ripeto - messe di profilo ed animate da un sapore più descrittivo e "cortese" [...] rinviano al rientro dell'artista dalla probabile parentesi napoletana» [P. LEONE DE CASTRIS, Gli affreschi dell'antico coro della chiesa di San Domenico, op. cit. (n. 10), p. 481]: questo il migliore punto di partenza per la nostra analisi stilistica (si veda infra nel testo).

${ }^{13}$ Ibidem, pp. 480-481.

${ }^{14}$ Massimo, Venanzio, Luciano, Comizio e Donato. Ibidem, p. 480.

${ }^{15}$ Ibidem, p. 481 .

${ }^{16}$ Per la zona in cui era originariamente situato il ciclo, preferiamo utilizzare il più specifico termine "abside" anziché "coro" (si vedano a proposito infra nel testo e la nota 49).

${ }^{17}$ Gli archi diaframma che scandiscono l'aula sono stati aggiunti in epoca successiva, forse quattrocentesca: P. LEONE DE CASTRIS, Gli affreschi del presunto Luca d'Atri. Chiesa di San Domenico. Teramo, in Teramo e la valle del Tordino, op. cit. (n. 11), pp. 429-439: 438 nota 1. Ringrazio Marco D'Attanasio per avermi fornita la fotografia riprodotta nel testo.

${ }^{18}$ Il più antico documento conservato riguardo la chiesa di Sant'Agostino sembra essere l'atto del 22 giugno 1358 edito in T. DI CRESCENZO, Elenco analitico dei documenti medievali (secc. X-XV) conservati presso l'Archivio dell'Arcidiocesi di Pescara-Penne, in M. Del Monte (ed.), Episcopati e monasteri a Penne e in Abruzzo (secc. XII-XIV). Esperienze storiografiche e storiche a confronto, Napoli, 2007, pp. 379-465: 408 doc. 112. Anche in questo edificio le pareti laterali sono scandite da monofore lanceolate chiuse forse in occasione della ristrutturazione barocca. Il finestrone absidale fu murato in epoca alta, con 


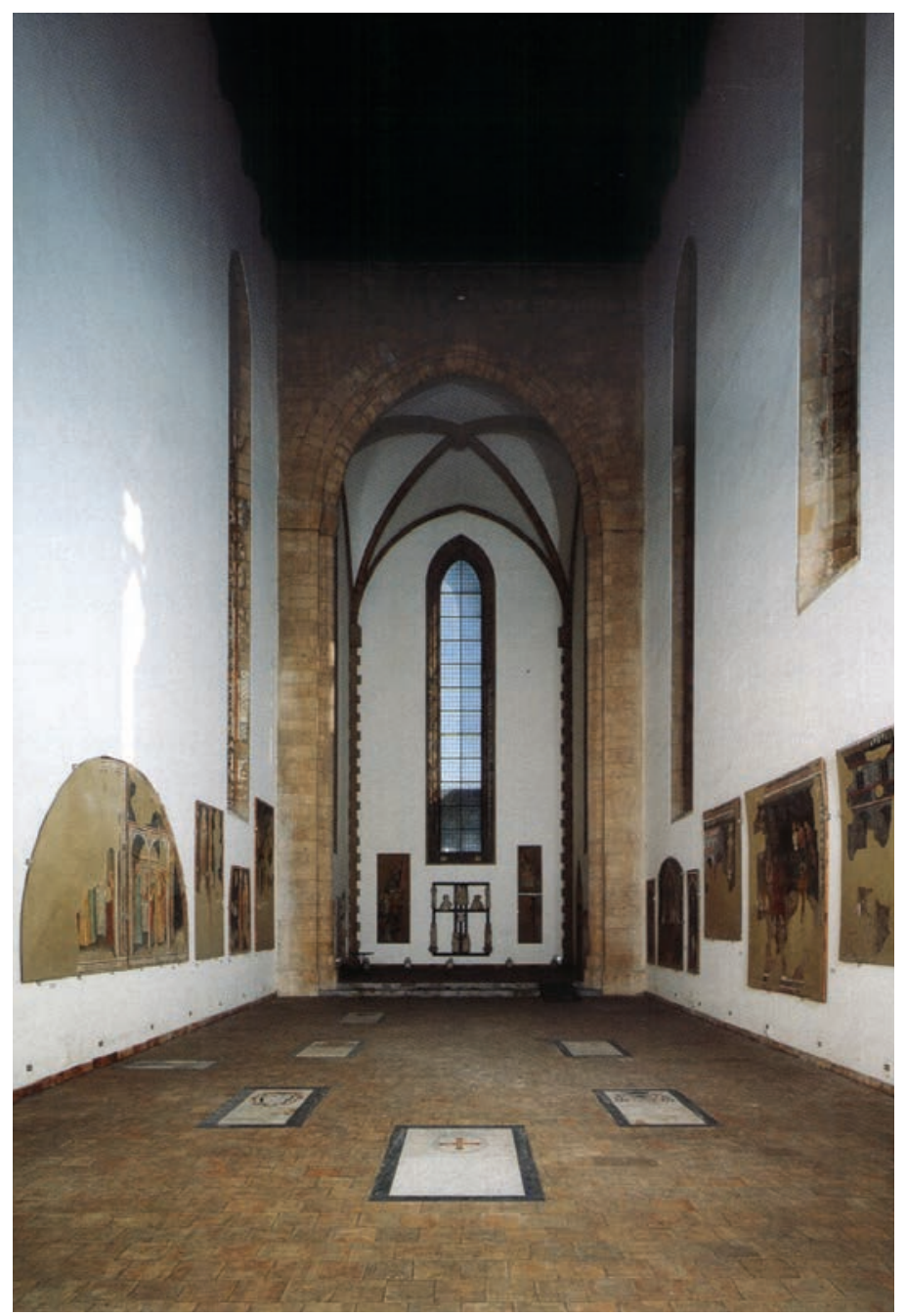

Fig. 4- Napoli, Castel Nuovo, Cappella Palatina, interno (da P. LEONE DE CASTRIS, Giotto a Napoli)

Cappella Palatina di Castel Nuovo (fig. 4), coincidenza che, come avremo modo di constatare più avanti, si rivela di una certa suggestione.

Osserviamo ciò che resta del ciclo. Volgendoci verso est, e lasciando idealmente alle nostre spalle l'ombrosa aula barocca, ecco sulla sinistra la scena della Strage degli Innocenti (Erode si affaccia da una loggia ordinando il massacro; al di sotto, una madre si scosta in preda al terrore, mentre una grossa mano afferra un piccolo braccio, fig. 5) e, assai meglio leggibile, quella della Presentazione al Tempio, dove la cerimonia si svolge, in un morbido alternarsi di tinte pastello, all'interno di una architettura cosmatesca di impronta masiana, notevole nella sua accurata ma poco profonda scansione spaziale (fig. 6). Quest'ultimo riquadro suscita interesse anche per via della presenza di una probabile committente laica - ritratta di profilo e in proporzioni non inferiori ai personaggi sacri - che sembra quasi partecipare al rito, con le mani giunte e il capo velato da una candida fascia che si avvolge attorno al collo e ricade su di una spalla. Nella parete di fondo campeggiano due lacerti purtroppo assai consunti: il primo, a sinistra, raffigura con tutta evidenza il Cristo tra i Dottori (Giuseppe e Maria tendono le braccia verso il bambino, affiancato da uno sbigottito sapiente che stringe in mano un filatterio coperto di caratteri pseudocu-

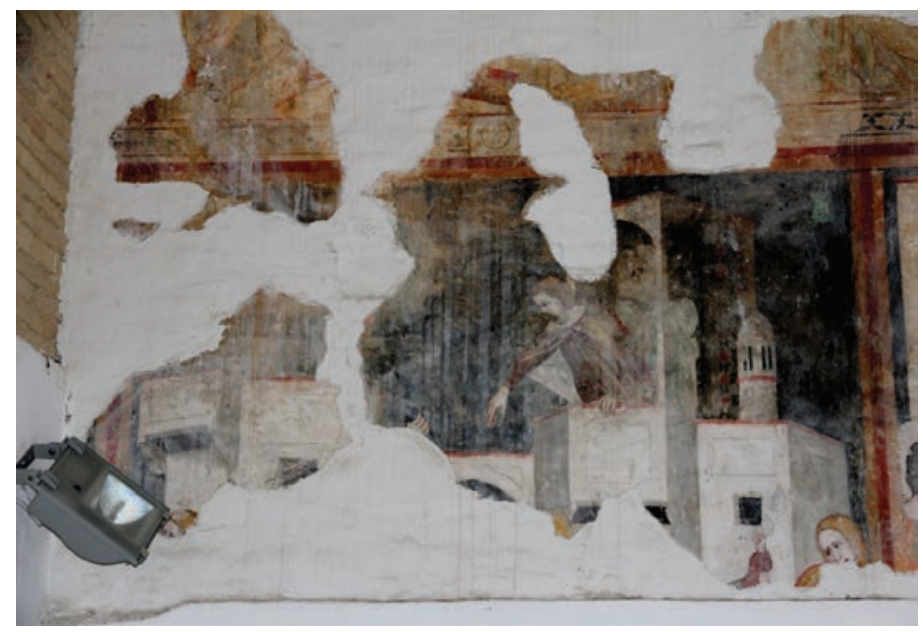

Fig. 5 - Penne, Chiesa di San Domenico, affreschi, Strage degli Innocenti

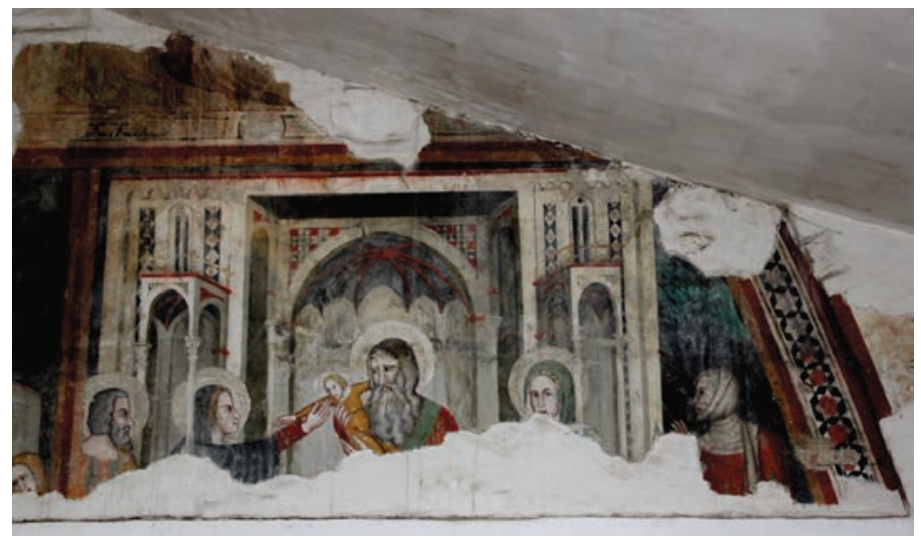

Fig. 6-Penne, Chiesa di San Domenico, affreschi, Presentazione al Tempio

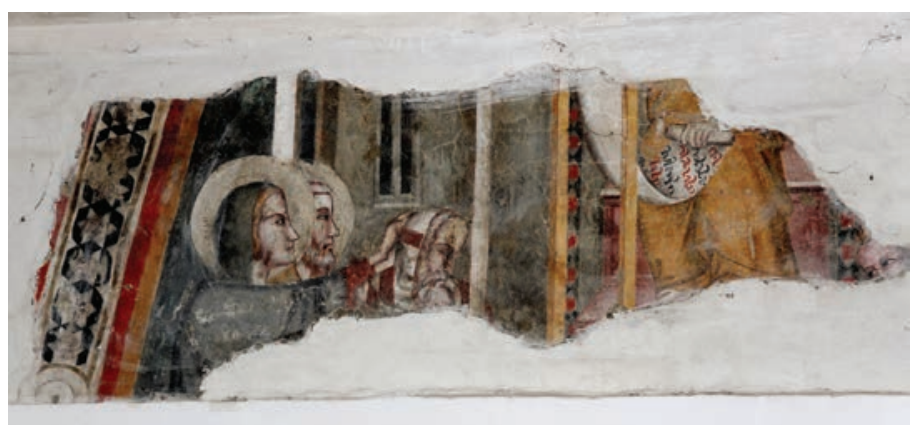

Fig. 7 - Penne, Chiesa di San Domenico, affreschi, Gesù tra i Dottori

fici, fig. 7); il secondo, all'estrema destra, un uomo di spalle che si affaccenda di fronte a una enigmatica struttura in legno, mentre un personaggio acefalo siede di profilo con una verga in mano, come nell'atto di ordinare qualcosa (fig. 8). Nella parete contigua, ci imbattiamo in un ampio lacerto comprendente alcune figurazioni molto frammentarie che ne circondano una più grande e meglio conservata, dove un giovane santo, dai capelli biondi e dal portamento elegante, fisiognomicamente individuato nel profilo inciso come un ritratto angioino, volge le mani giunte verso un cielo azzurro, interrotto da sagome di alberi e dal balenare inaspettato di una manus Dei, mentre un secondo personaggio, sulla destra, si copre il volto come per ripararsi da una improvvisa folata di vento, che muove leggermente le fronde e fa vacillare la fiamma di una torcia tenuta in mano dalla

tutta evidenza per permettere la messa in opera del misterioso affresco della Crocifissione (per il quale si veda infra la nota 57). Una tipologia simile è altresì ravvisabile in San Giovanni Evangelista, anche questa di origine trecentesca e poi fatta oggetto delle consuete risistemazioni barocche durante il XVIII secolo. 


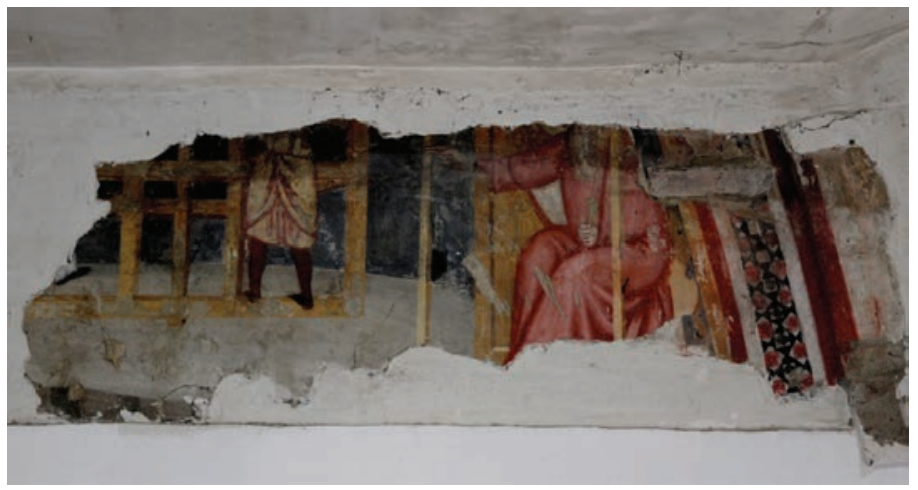

Fig. 8 - Penne, Chiesa di San Domenico, affreschi, Costruzione dell'Arca

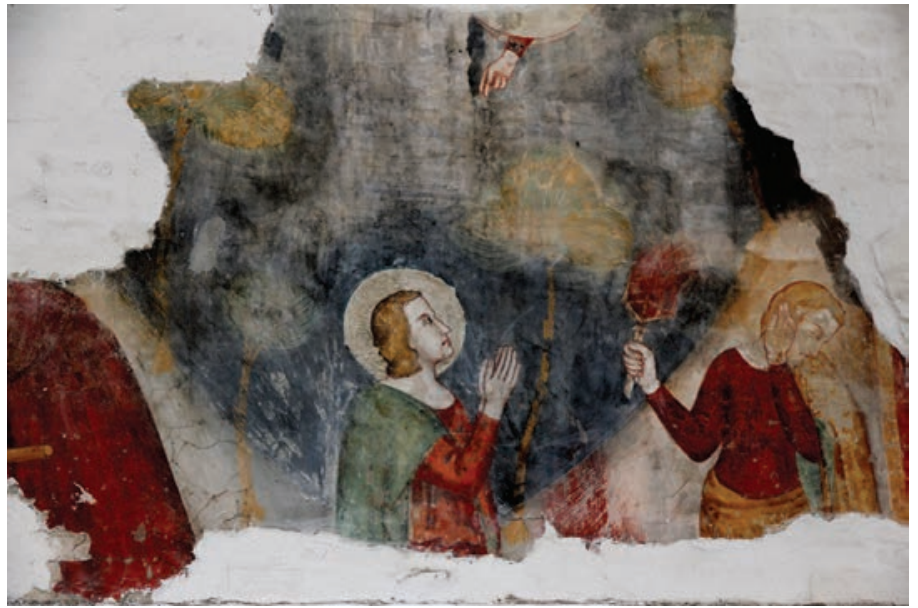

Fig. 10 - Penne, Chiesa di San Domenico, affreschi, Martirio di San Giovanni Evangelista

misteriosa comparsa (fig. 10). Ci troviamo di fronte alle due scene che Leone de Castris ha in via ipotetica interpretate come episodi successivi di un martirio, dalla preparazione alla sua esecuzione. Come vedremo immediatamente, non solo l'ipotesi è giusta, almeno per la seconda scena, ma ci dà la possibilità di spingerci ancora più avanti e proporre per il martire un nome preciso.

Prima, però, tentiamo di ricostruire per sommi capi il programma iconografico. Le tre scene a sinistra, la cui identificazione con la Strage degli Innocenti, la Presentazione al Tempio e il Cristo tra i Dottori è chiarissima, fanno pensare allo svolgersi di un ciclo di storie del Nuovo Testamento. La direzione di lettura sembra essere quella sinistra-destra, e se, come convincentemente ipotizzato, il lunettone recava in origine una Natività (si scorgono soltanto profili di rocce), gli episodi dovevano cronologicamente disporsi su più registri secondo la consueta disposizione alto-basso e sinistra-destra. Più problematici da inserire, però, sono gli altri lacerti, certamente non identificabili con scene neotestamentarie. Come decifrarli?

Il frammento con lo strano traliccio ligneo (fig. 8) può essere utilmente messo a confronto con la scena della Costruzione dell'Arca nella Bibbia Hamilton (Berlino, Staatliche Museen, Kupferstichkabinett, ms. 78 E 3, f. 4r, fig. 9): non sono soltanto le linee generali a corrispondere, ma persino alcuni dettagli: si noti ad esempio la sorprendente

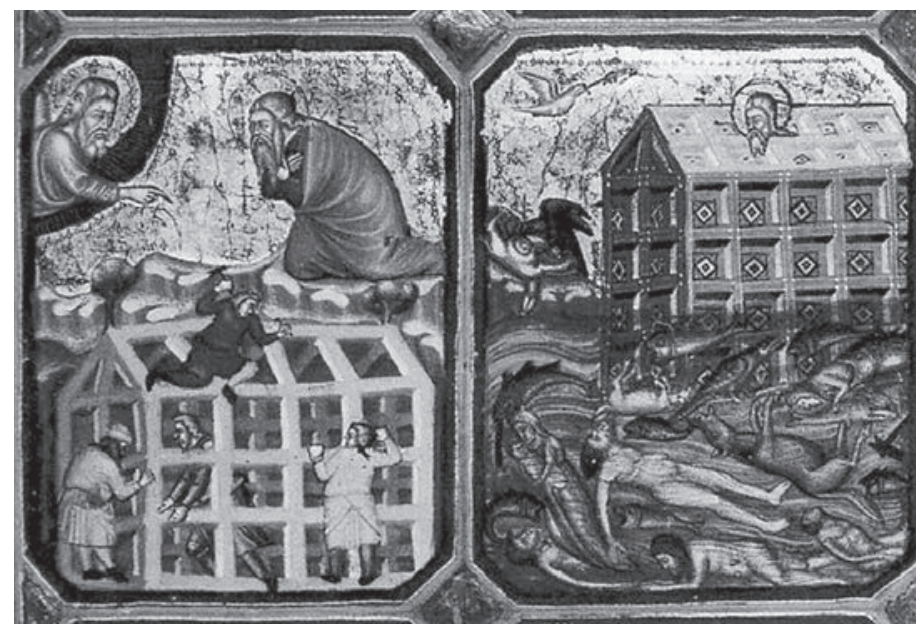

Fig. 9 - Berlino, Staatliche Museen, Kupferstichkabinett, ms. 78 E 3 (Bibbia Hamilton), f. 4r, dettaglio, Costruzione dell'Arca (da BRÄM, Neapolitanische Bilderbibeln des Trecento)

somiglianza del panneggio nell'abito dell'uomo che ci dà le spalle, così notevole da spingerci a postulare l'esistenza di un prototipo comune. La figura di Noè con la verga in mano è invece presente, seppure in posizione diversa, nella Bibbia di Vienna (Österreichische Nationalbibliothek, codex 1191, $\mathrm{f}$. $6 r)^{19}$. Lasciando per il momento da parte ipotesi più o meno ardite su possibili fonti monumentali o sulla circolazione di modelli precisi, la lettura della scena misteriosa aggiunge alla nostra analisi un elemento in più, e d'importanza per nulla secondaria: oltre al ciclo evangelico, le pareti dovevano ospitarne anche uno dedicato all'Antico Testamento.

Spostiamoci sulla parete meridionale. La grande scena di "martirio" (fig. 10) non ha certo nulla a che vedere con la Genesi, e la sua contiguità con l'episodio dell'Arca di Noè è quindi da escludere. La posizione del protagonista, che a mani giunte implora l'intervento divino, rende l'ipotesi di Leone de Castris assolutamente credibile. Ma di quale santo si tratta? L'aspetto giovanile e finanche azzimato potrebbe calzar bene a tutta una serie di martiri più o meno famosi, e la mancanza di attributi risolutivi non fa che accrescere la nostra perplessità. Ma concentriamoci meglio: abbiamo notato in precedenza che la scena sembra animata da un forte vento, che quasi spegne la torcia del probabile aguzzino. Ora, se cerchiamo di ricostruire e integrare la parte inferiore del riquadro, irrimediabilmente perduto, potremmo in effetti supporre che il santo si trovi al di sopra di una graticola, o comunque di un fuoco, estinto dalla miracolosa contingenza atmosferica. Se al posto della graticola (difficile identificare il giovane con il diacono Lorenzo) immaginiamo un calderone pieno d'olio bollente, verrebbe a formarsi l'immagine del supplizio dell'evangelista Giovanni sul Celio - nel luogo in cui, secondo la tradizione, sarà edificata la chiesa di San Giovanni in Òleo - prima del suo esilio nell'isola di Patmos ${ }^{20}$, teatro di quelle visioni apocalittiche così care alla cultura religiosa angioina. Un confronto iconografico di àmbito napoletano potrebbe essere istituito ancora una volta con la Bibbia Hamilton, dove, però, Giovanni è nudo (fig. 11) ${ }^{21}$. Resta comunque significativa la somiglianza nella posa del

${ }^{19}$ Sulle Bibbie angioine, si faccia naturalmente riferimento a A. BRÄM, Neapolitanische Bilderbibeln des Trecento. Anjou-Buchmalerei von Robert dem Weisen bis zu Johanna I, Wiesbaden, 2007, con prezioso volume di tavole.

${ }^{20}$ P.C. LANDUCCI, F. SPADAFORA, M.C. CELLETTI, ad vocem Giovanni Evangelista, in Bibliotheca Sanctorum, VI, Roma, 1966 (e rist. 1988), coll. 757-797: $763,786$.

${ }^{21}$ A f. 453 r. 


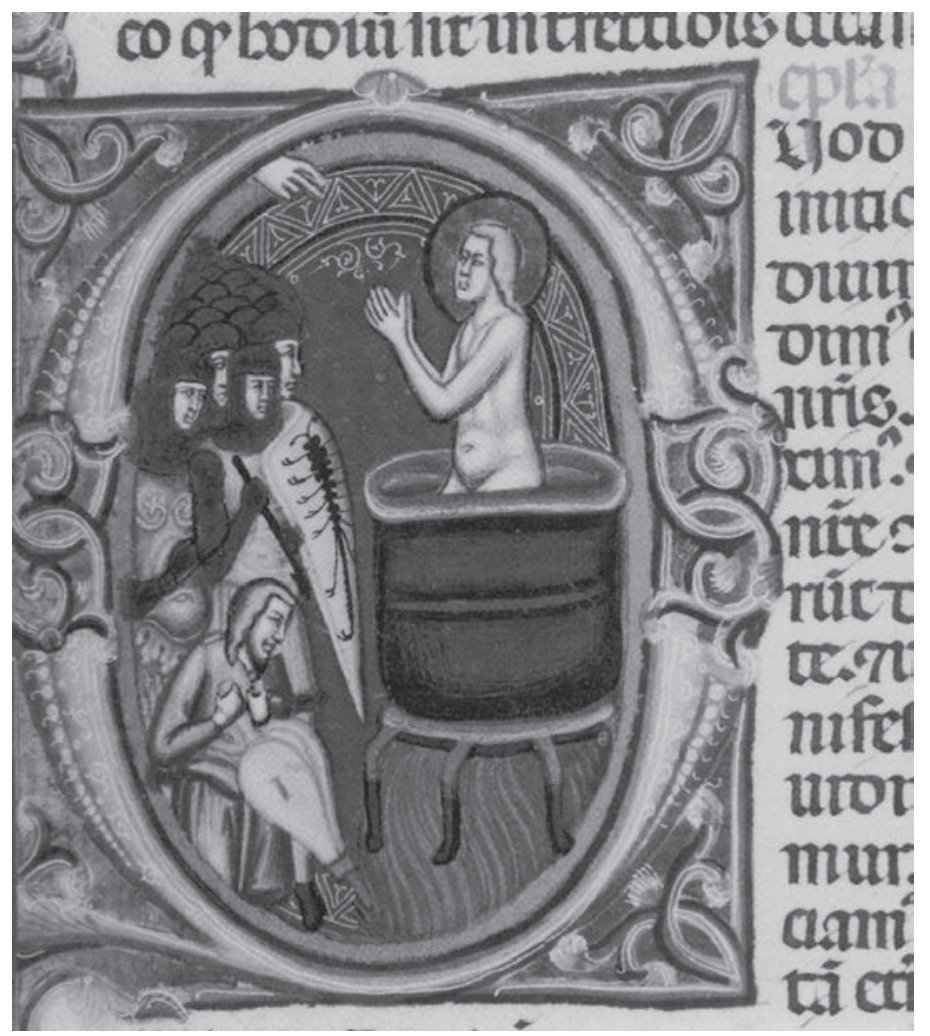

Fig. 11 - Berlino, Staatliche Museen, Kupferstichkabinett, ms. 78 E 3 (Bibbia Hamilton), f. 453r, dettaglio, Martirio di San Giovanni Evangelista (da BRAM, Neapolitanische Bilderbibeln des Trecento)

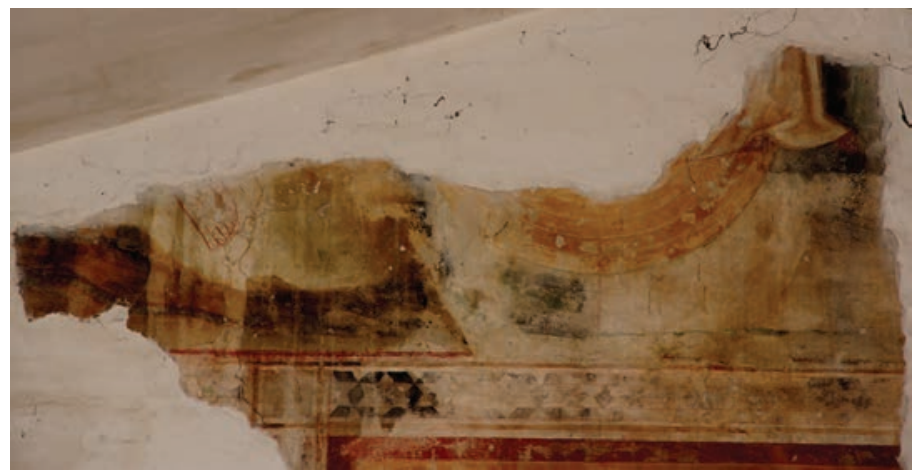

Fig. 14 -Penne, Chiesa di San Domenico, affreschi, Creazione del Mondo e Creazione di Adamo ed Eva

santo in preghiera, dall'aspetto giovanile, rivolto, occhi al cielo, verso la manus Dei. L'ipotesi potrebbe essere corroborata dall'esiziale frammento che, proseguendo la lettura secondo la direzione canonica, affianca a destra la grande scena e che reca l'immagine di una sorta di ventaglio di lame appuntite (fig. 12): l'ala spinosa di un mostro? oppure, più plausibilmente, le foglie pennate della palma sotto la quale Giovanni siede nel primo episodio dell'Apocalisse così come narrata ad esempio nelle celebri tavole di Stoccarda (fig. 13 $)^{22}$ ? In tal caso, oltre all'Antico e al Nuovo Testamento, avremmo anche un ciclo giovanneo e apocalittico.

Nel registro superiore, pur tra le vaste e definitive lacune, sono ben visibili dei cerchi concentrici, parzialmente coperti da un mantello, e un piede nudo su di una roccia frastagliata (fig. 14). Se l'idea di vedere nel lunettone le scene

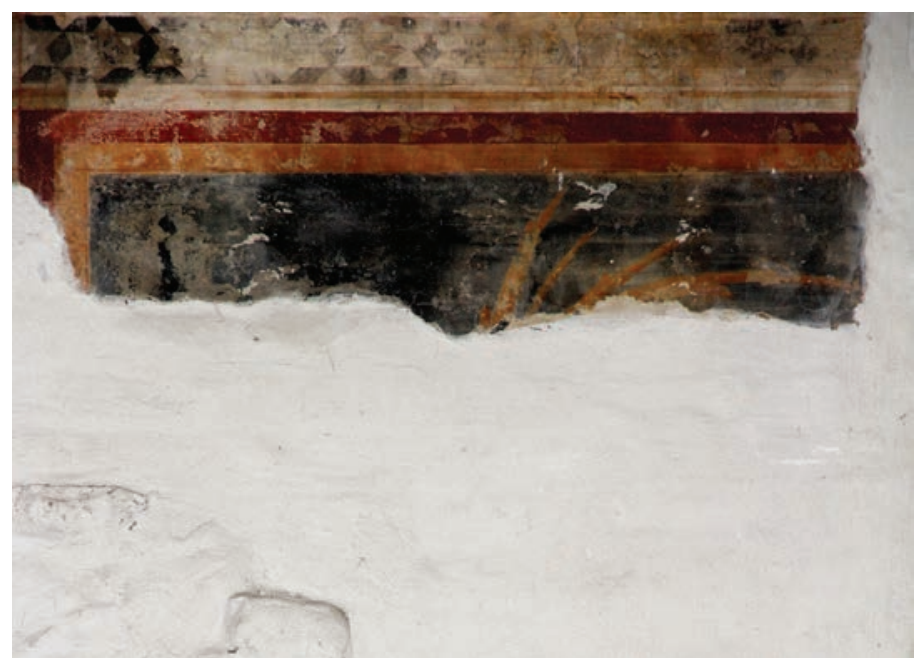

Fig. 12 - Penne, Chiesa di San Domenico, affreschi, San Giovanni a Patmos (?)

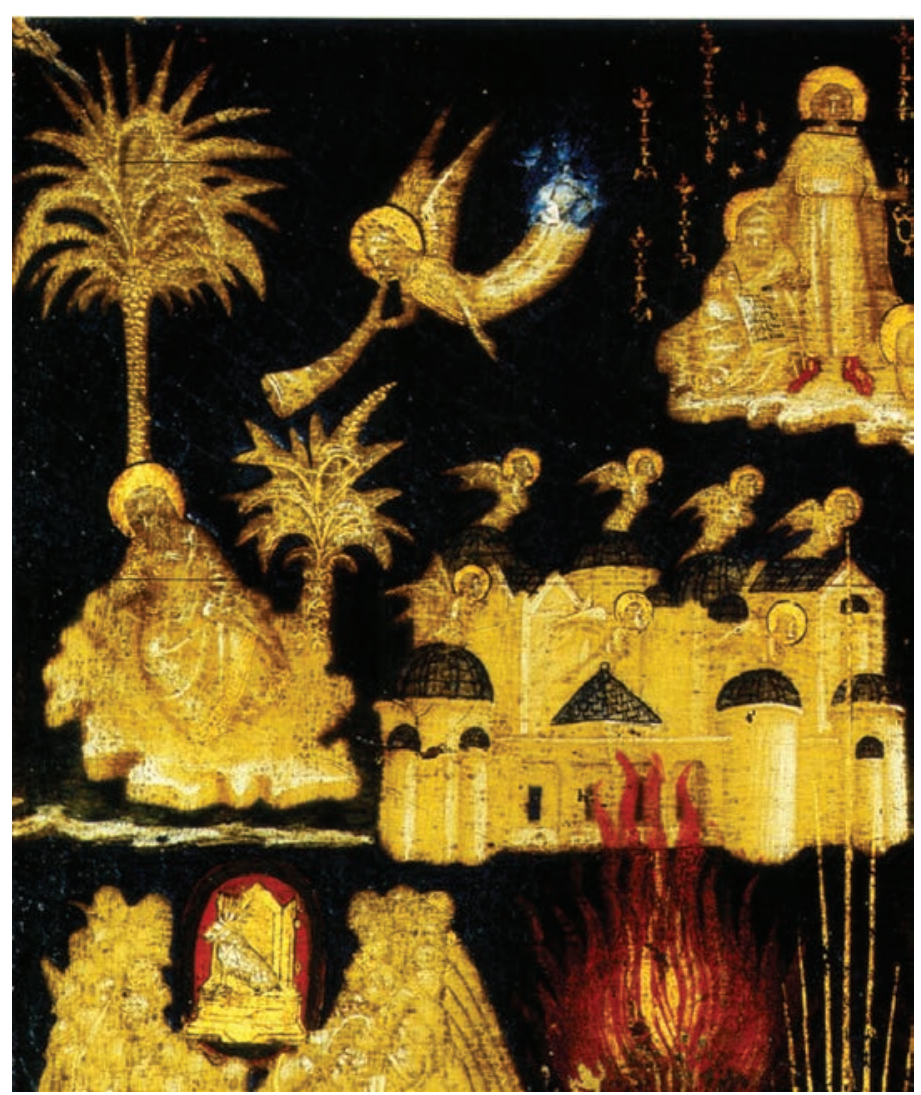

Fig. 13 - Stoccarda, Staatsgalerie, Storie dell'Apocalisse, dettaglio, San Giovanni a Patmos (da P. LEONE DE CASTRIS, Giotto a Napoli)

dell'Apocalisse è tentante (e il piede farebbe pensare proprio alla canonica immagine di san Giovanni a Patmos, magari circondato da sintetiche visioni come nella Cappella Peruz$\mathrm{zi}$ ), anche in questo caso ci soccorrono le Bibbie angioine: un confronto tra la Creazione del Mondo e quella di Adamo ed Eva nella già citata Bibbia Hamilton (fig. 15$)^{23}$ dimostra chiaramente che ci troviamo dinanzi a episodi della Genesi che precedono quello dell'Arca: il mantello appartiene al Creatore, che come una sorta di chimico sembra far reagire gli elementi agitandoli con una bacchetta; il piede, ad Adamo

22 P. LEONE DE CASTRIS, Giotto a Napoli, Napoli, 2006, pp. 128-129 e 160 nota 40 (con bibliografia precedente) e ottime e grandi riproduzioni alle pp. 134-135 e 138-139; A. TOMEI, I pannelli dell'Apocalisse di Stoccarda e altre visioni angioine, in Ikon, 6, 2013, pp. 65-78.

${ }^{23}$ Ma si veda anche il già citato f. 6r della Bibbia di Vienna. 


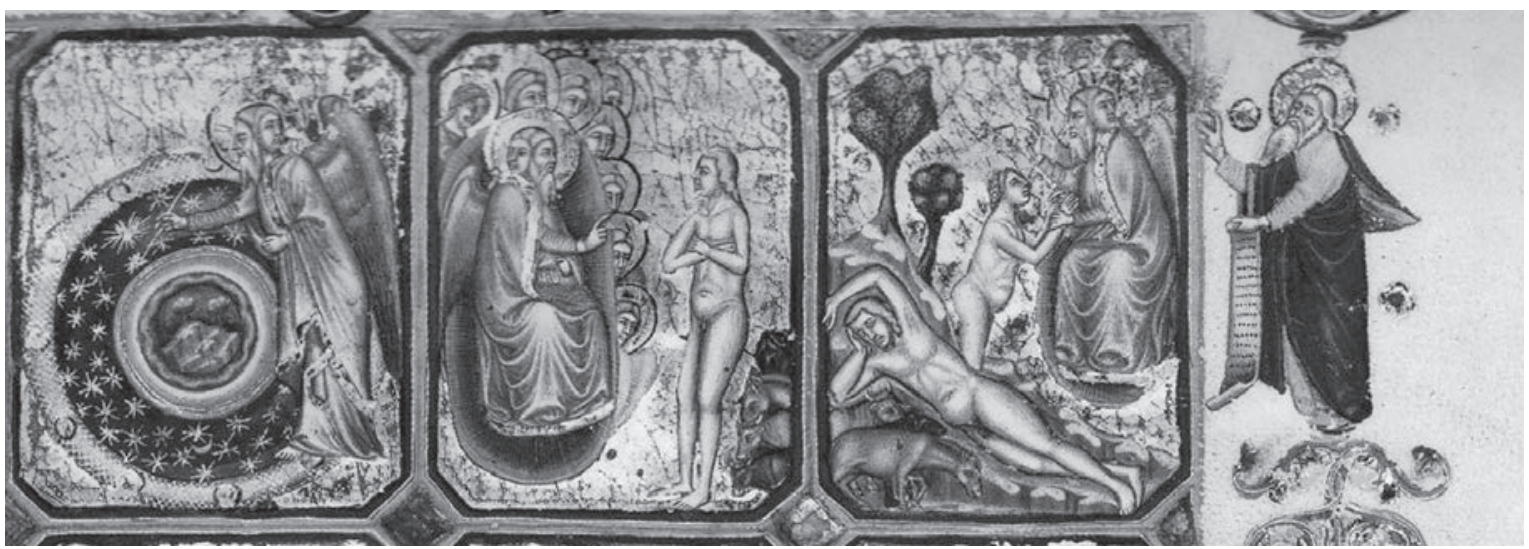

Fig. 15 - Berlino, Staatliche Museen, Kupferstichkabinett, ms. 78 E 3 (Bibbia Hamilton), f. 4r, dettaglio, Creazione del Mondo e Creazione di Adamo ed Eva (da BRÄM, Neapolitanische Bilderbibeln des Trecento)

addormentato. La lettura, in questo caso, doveva dunque svolgersi da sinistra a destra.

Riassumendo: le pareti dell'abside erano completamente coperte di affreschi con storie dell'Antico e del Nuovo Testamento, e, se la più problematica ipotesi ha, come ci sembra, un margine di verisimiglianza, la leggenda di san Giovanni Evangelista e le visioni dell'Apocalisse. Una storia sacra universale, che richiama ad esempio quella narrata da Giusto de' Menabuoi nel Battistero di Padova ${ }^{24}$, e nella quale è facile immaginare contrapposti gli episodi chiave, in un gioco di allusioni e rimandi: alla Creazione corrispondeva forse la Natività, e la Crocifissione doveva fronteggiare l'Apocalisse. Nella parete di fondo, la monofora centrale segnava una cesura tra le scene neotestamentarie, sulla sinistra, e le storie dell'Antico Testamento e di san Giovanni, sulla destra.

A questi frammenti, già in parte editi, possiamo aggiungerne un altro, assai ampio e di una certa qualità ma finora ignoto anche alla letteratura locale, forse perché situato in un diverso ambiente e mai fatto oggetto di valorizzazione o di semplice restauro conservativo. Come abbiamo visto, il grande cantiere settecentesco lasciò pressoché intatto il paramento esterno. Non solo: il "guscio" barocco che rimodella l'aula non poggia sull'antica muratura perimetrale, ma su un'altra cortina a mattoni realizzata in epoca successiva. Tra i due muri corre una stretta intercapedine che, nel fianco settentrionale della chiesa, attraverso una porticina che si apre tra il campanile e il deambulatorio del secondo ordine del chiostro, è in parte percorribile e adibita a magazzino ${ }^{25}$. In corrispondenza dell'antico arco absidale si conserva un ampio lacerto su tre registri, posto grossomodo al livello dei frammenti esaminati: nella parte alta, una grande ala coperta di piume multicolori (fig. 16); in basso, una figura femminile all'interno di una struttura architettonica assai elaborata (fig. 17); al di sotto di questa, una sorta di stemma frammentario e di difficilissima lettura (fig. 18). Ė pressochéautomatico vedere nei primi due ciò che resta di un'Annunciazione e, forse, $\mathrm{di}$ una Visitazione (scontato antefatto alla probabile Natività); più difficile è purtroppo decifrare le spoglie di quello che sembrerebbe essere uno stemma, racchiuso in uno scudo francese antico appuntato. Costanza Cipollaro suggerisce la presenza di un gonfalone rosso a due punte, sovrastato a destra da una

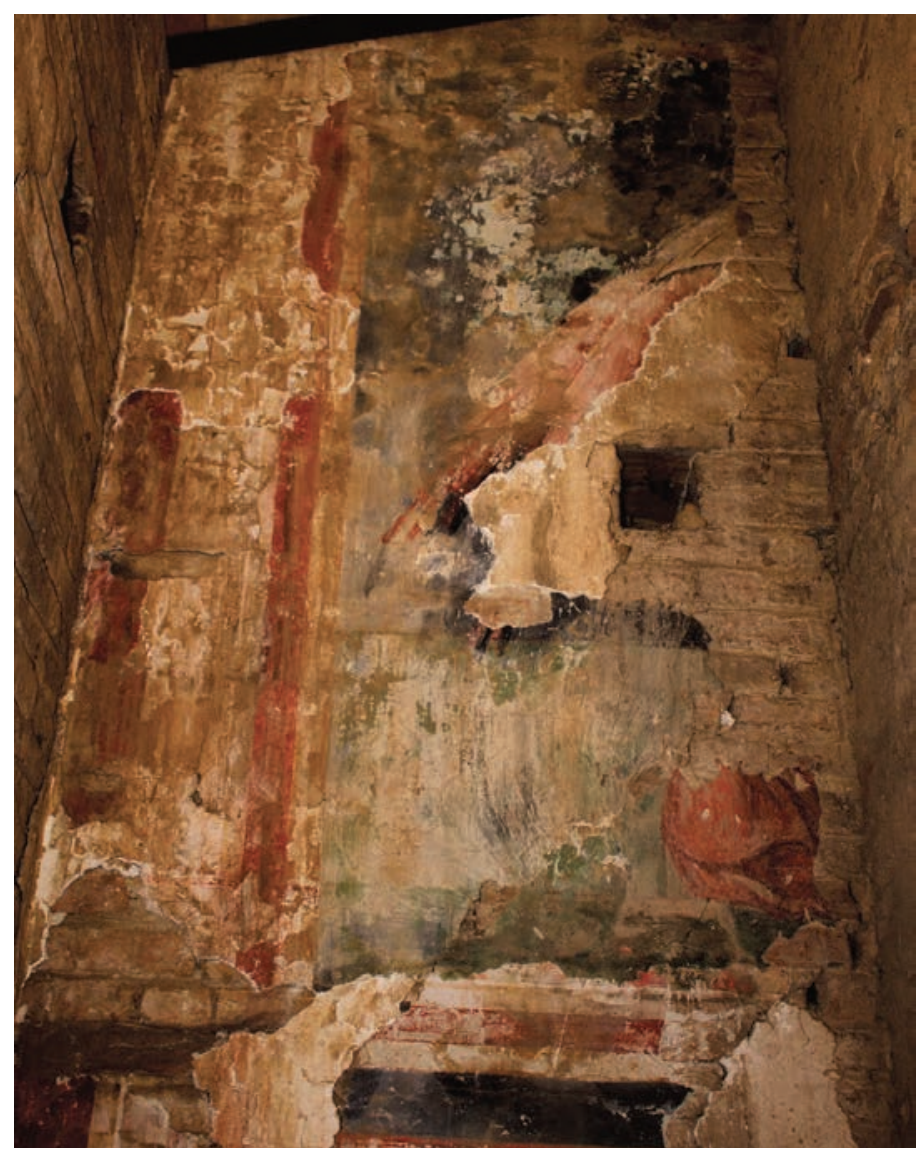

Fig. 16 - Penne, Chiesa di San Domenico, affreschi, Annunciazione

piccola croce potenziata, simile a quella dell'Ordine del Santo Sepolcro di Gerusalemme. Manca però qualsiasi elemento araldico utile a collegare l'arme, se di arme si tratta, ad una famiglia conosciuta ${ }^{26}$. Tuttavia, l'ipotetica presenza di una committente laica lascia immaginare che nella realizzazione dell'opera dovettero partecipare membri dell'aristocrazia o di una qualche confraternita: il velo indossato dalla figura muliebre che assiste alla Presentazione al Tempio sembra essere molto simile a quello dell'ordine secolare femminile domenicano (lo si confronti ad esempio con l'abito di santa Caterina nel dipinto celeberrimo di Andrea Vanni in San

\footnotetext{
${ }^{24}$ Su Giusto è in corso di preparazione, da parte di chi scrive, una monografia critica. Sul Battistero, si può ancora fare riferimento a A.M. Spiazzi (ed.), Giusto de' Menabuoi nel Battistero di Padova, Trieste, 1989.

${ }^{25}$ Ringrazio il parroco, don Giorgio Moriconi, per aver agevolato, con pazienza ed entusiasmo, le ricerche in loco.

${ }^{26}$ Comunicazione scritta.
} 


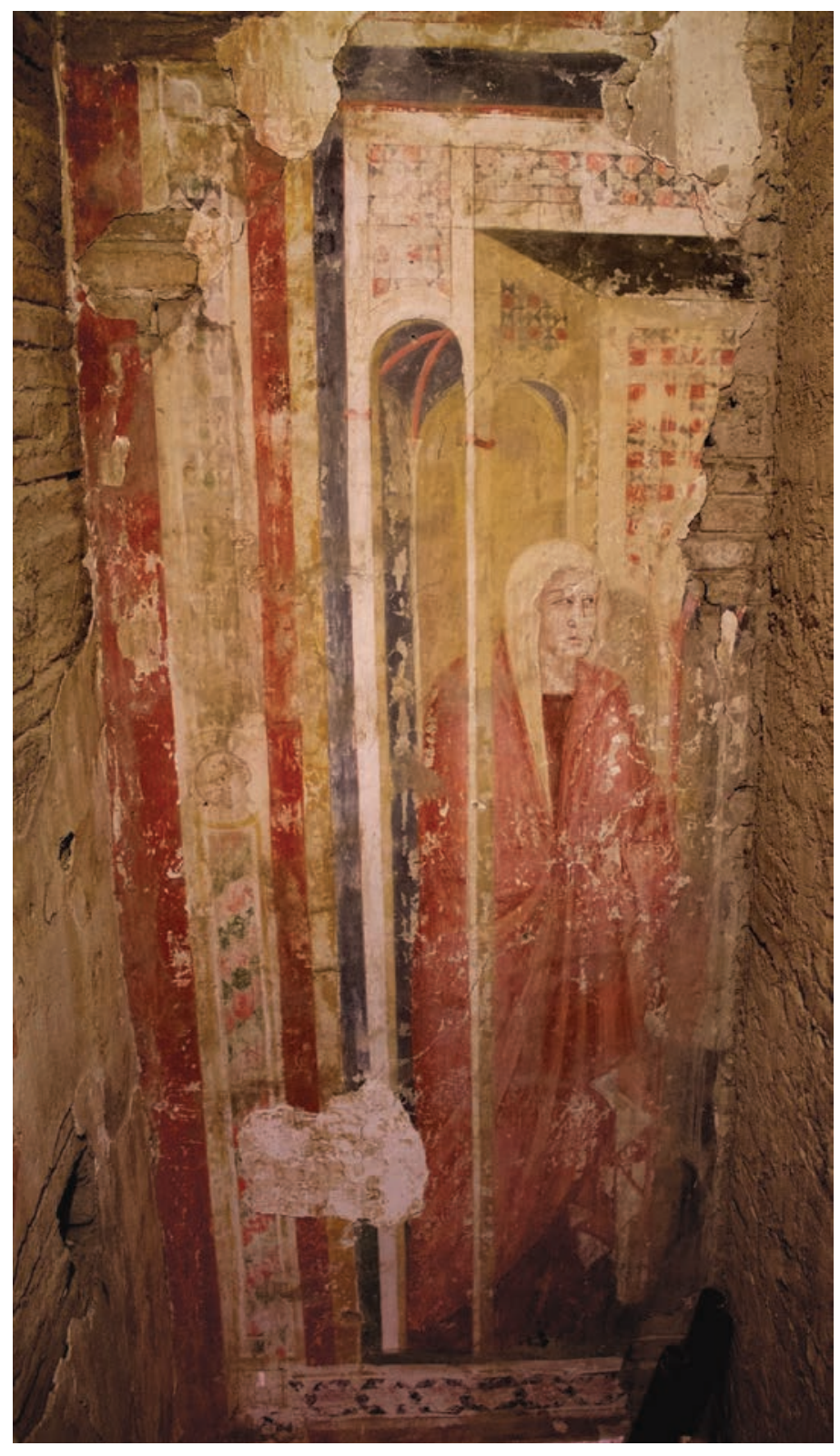

Fig. 17 - Penne, Chiesa di San Domenico, affreschi, Visitazione (?)

Domenico a Siena), ma manca purtroppo il mantello nero che ci permetterebbe di avanzare questa identificazione senza alcun dubbio.

3. Cerchiamo ora di definire le coordinate stilistiche degli affreschi. La pista napoletana suggerita da Leone de Castris è senz'altro quella che può essere percorsa con maggior profitto, diluendo ulteriormente le ipotetiche componenti

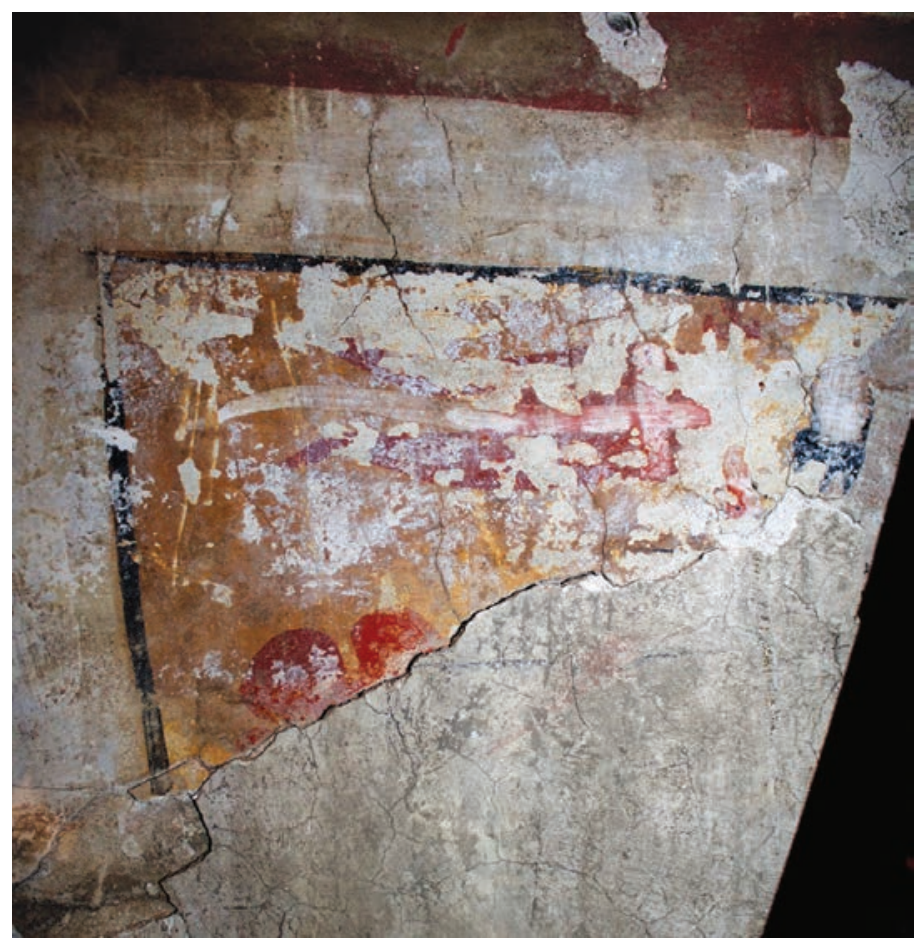

Fig. 18 - Penne, Chiesa di San Domenico, affreschi, stemma (?)

"marchigiane" e riminesi, in questo caso davvero impercepibili, e svincolando ciò che resta del ciclo da altri, significativi numeri del cosiddetto Maestro di Offida, il cui eterogeneo catalogo, pur nelle differenze imputabili alla varietà delle botteghe operanti negli Abruzzi (che, specie nel periodo tardogotico, mostreranno tangenze e mescolanze imprevedute), è purtroppo soggetto a più interpretazioni e dubbi riguardo anche l'ipotetica collocazione cronologica di molti dei dipinti. Ci basti un confronto tra la nostra Presentazione al Tempio (fig. 6) e uno qualsiasi dei murali della Chiesa Inferiore di Santa Maria della Rocca ad Offida (fig. 19), luminoso name-piece dello squisito maestro, databile agli anni Sessanta ${ }^{27}$, per renderci conto delle grandi differenze tra le due imprese: gli affreschi di Penne, infatti, mancano del tutto di quella dolcezza quasi estenuata che, presupposti "padani" a parte, in alcuni dettagli sembrerebbe persino rivelare un primo sentore di certi modi più specificamente felsinei, simili ad esempio a quelli di Andrea da Bologna, cui non a caso furono attribuiti ${ }^{28}$, che si faranno preponderanti nel settentrione del Regno a partire dall'ottavo decennio con la figura di Antonio da Atri e della sua bottega tra la città natale e L'Aquila ${ }^{29}$, fino agli echi ancora evidenti cinquanta anni dopo nel Maestro di Loreto Aprutino ${ }^{30}$, che a capo di una bottega composita elaborerà in maniera originalissima le fonti

\footnotetext{
${ }_{27}$ Tra il 1361 e il 1367: P. LEONE DE CASTRIS, Appendice. Pittura del Trecento nell'Abruzzo Teramano, op. cit. (n. 11), p. 443.

${ }_{28}^{28}$ B. BERENSON, Italian Pictures of the Renaissance, Oxford, 1932, p. 11. Si veda anche F. BOLOGNA, P. LEONE DE CASTRIS, Percorso del Maestro di Offida, op. cit. (n. 11), p. 300 nota 17.

${ }^{29}$ Su Antonio da Atri: P. LEONE DE CASTRIS, Appendice. Pittura del Trecento nell'Abruzzo Teramano, op. cit. (n. 11), p. 448 (con bibliografia pregressa a p. 453 nota 54); C. PASQUALETTI, Ascendenze emiliano-adriatiche nella pittura abruzzese dell'ultimo quarto del Trecento: nuovi affreschi di Antonio d'Atri nella chiesa di San Domenico all'Aquila, in Prospettiva, 2009, 133, pp. 46-68. Nel ciclo aquilano di San Domenico sono straordinariamente evidenti caratteri bolognesi che si direbbero mediati dalla miniatura: basti osservare il volto del suonatore di viella nella scena della Danza di Salomè e metterlo a confronto ad esempio con il contadino che cava le rape nella raffigurazione del mese di Ottobre nel celebre Officium Beatae Mariae Virginis di Forlì (Biblioteca Comunale "Aurelio Saffi", ms. 853).

$3^{\circ}$ Sul Maestro di Loreto Aprutino si vedano almeno, con bibliografia precedente: C. PASQUALETTI, Per la pittura tardogotica ai confini settentrionali del Regno di Napoli: sulle tracce del "Maestro del Giudizio di Loreto Aprutino" - 1 e 2, in Prospettiva, 2003, 109, pp. 2-26 e 2005, 117-118, pp. 63-99; EAD., Angeli ad affresco del portale maggiore e affreschi della controfacciata occidentale. Cattedrale. Teramo, in Teramo e la valle del Tordino, op. cit. (n. 11), pp. 309-311; EAD., Annunciazione e altri affreschi. Chiesa di San Domenico. Teramo, ivi, pp. 454-46o; EAD., Affreschi del Maestro del Giudizio di Loreto Aprutino. Chiesa di Santa Maria de Erulis. Ripattoni, ivi, pp. 461-462; F. MANZARI, Pittori e miniatori tardogotici tra Marche e Abruzzo: un messale miniato destinato a
} 

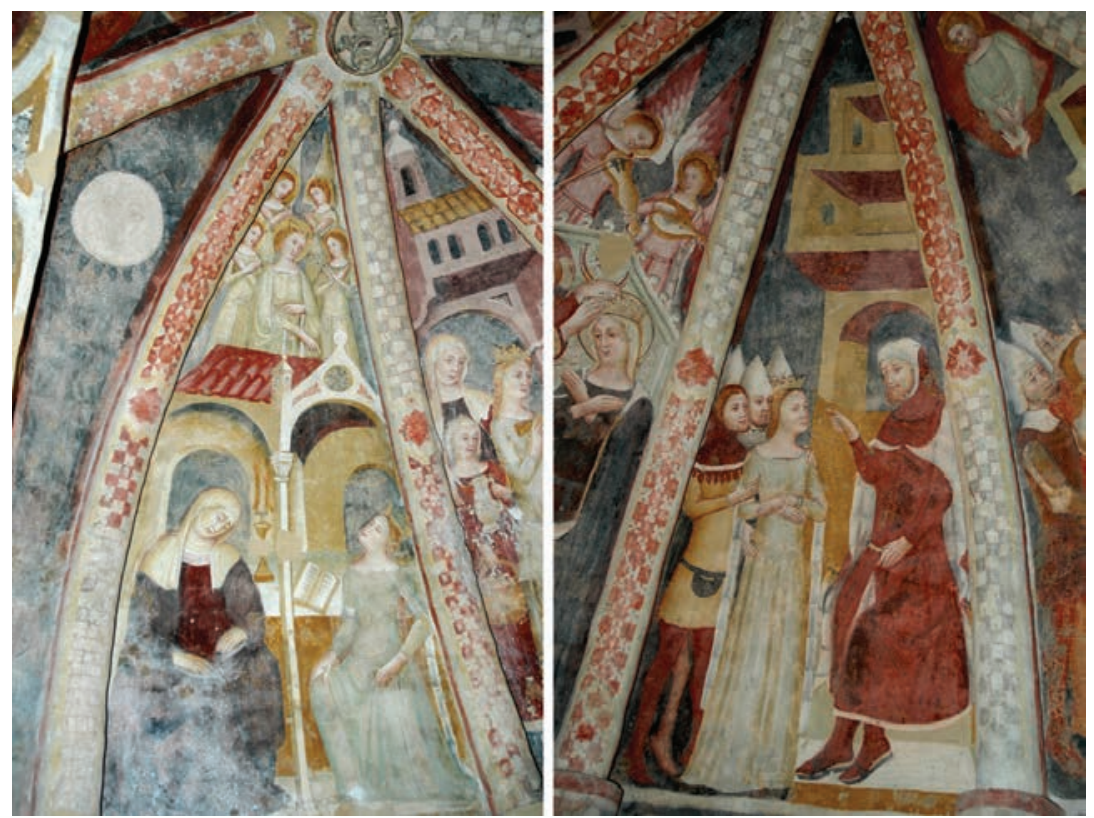

Fig. 19 - Offida, Santa Maria della Rocca, affreschi, Storie di Santa Caterina

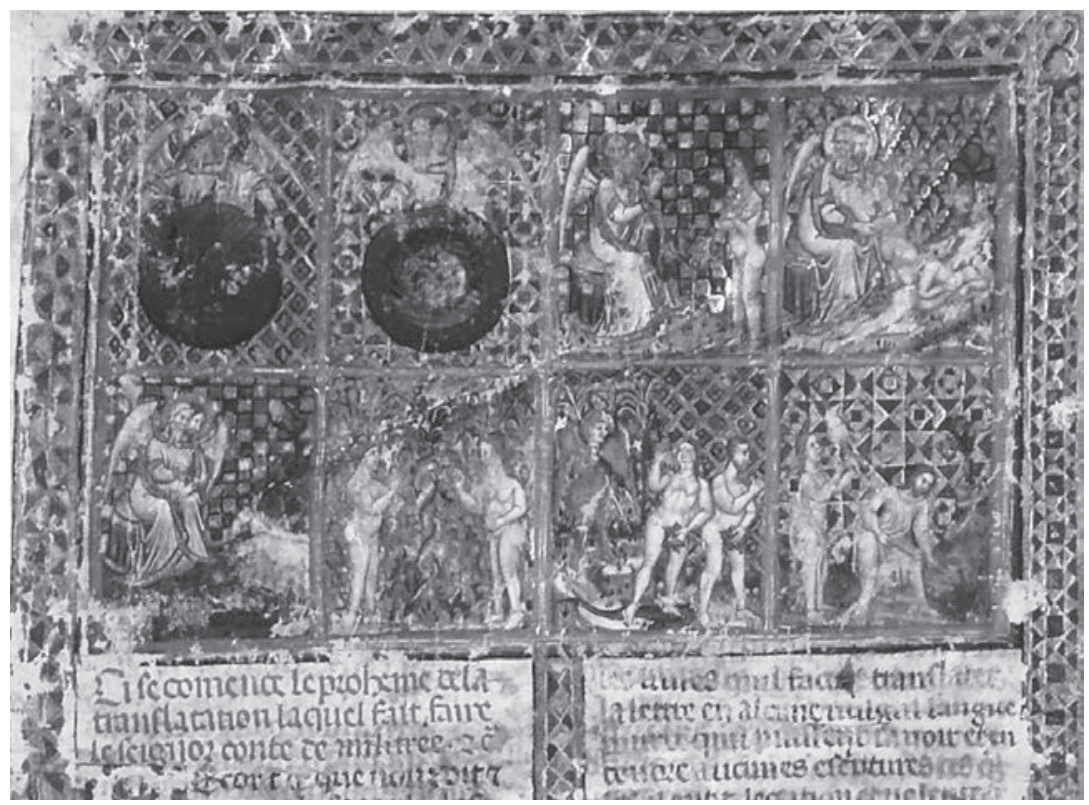

Fig. 21 - Parigi, Bibliothèque Nationale, ms. fr. 688 (Amato da Montecassino, Historia Normannorum), f. $1 r$, dettaglio (da J. KUJAWINSSKI, Alla ricerca del volgarizzamento...)

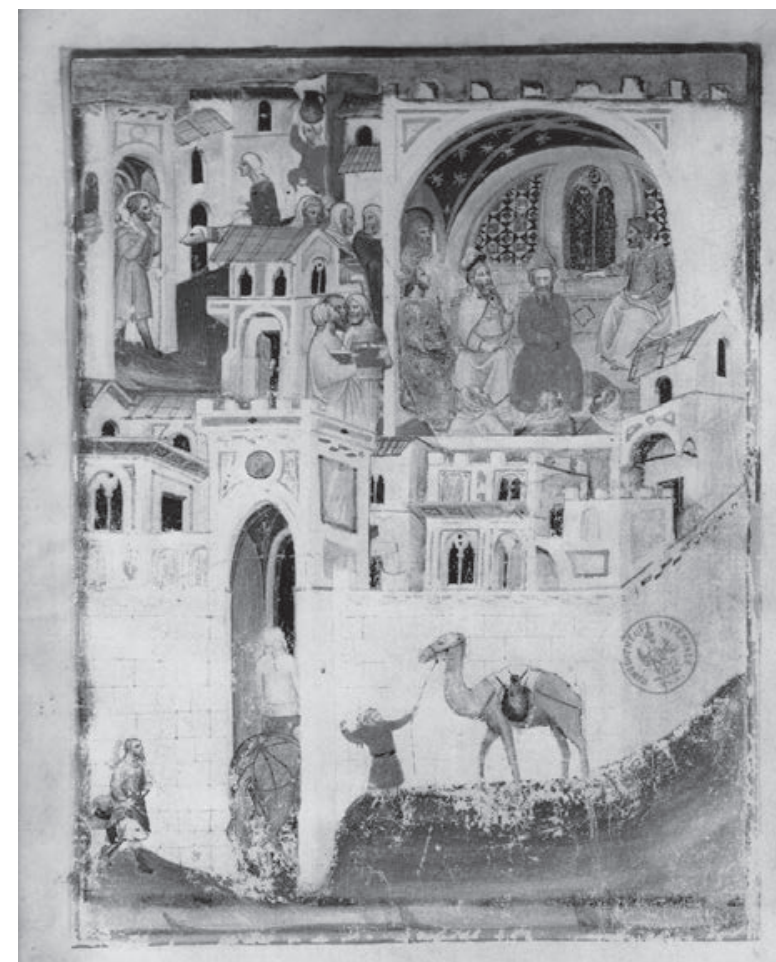

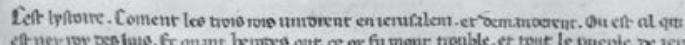

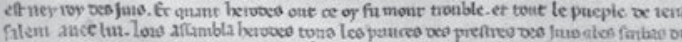

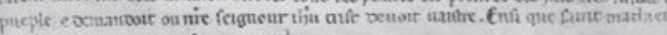
colit en fon enangrile ve fenut capitler

Fig. 20 - Parigi, Bibliothèque Nationale de France, Ms. fr. 9561 (Bible moralisée), f. 135v (da BRÄM, Neapolitanische Bilderbibeln des Trecento)

ancora una volta ricorda da vicino la decorazione libraria bolognese.

Del resto, già i confronti iconografici con le miniature angioine ci hanno parlato con chiarezza di uno stretto, inequivocabile rapporto con la capitale del Regno. Ma potremmo continuare: un altro suggestivo riferimento può essere ad esempio rintracciato nella Bible Moralisée di Parigi (fig. $20)^{31}$, dove ricorrono motivi ornamentali di gusto cosmatesco assai peculiari e molto vicini a quelli evidenti nei lacerti esaminati (soprattutto nelle bordure dei lunettoni, o nella scena della Presentazione al Tempio), nonché una simile scansione spaziale, giocata su edifici poco profondi, coperti da volte costolonate e rigogliosi di mosaici geometrici ${ }^{32}$. La cortesia di Francesca Manzari ci permette di aggiungere a questa breve casistica la pagina d'incipit del volgarizzamento della Historia Normannorum di Amato da Montecassino (Parigi, Bibliothèque Nationale, ms. fr. 688) ${ }^{33}$, contenente, guarda caso, scene della Genesi (fig. 21). Il riquadro con la raffigurazione della Nascita di Eva reca sullo sfondo un tappeto

Offida, in Civiltà urbana e committenze artistiche, op. cit. (n. 11), pp. 161-188; A. TOMEI, Qualche precisazione sugli affreschi tardogotici in Santa Maria della Rocca ad Offida, ivi, pp. 327-351.

${ }^{31}$ Bibliothèque Nationale, ms. fr. 9561.

${ }^{32}$ Citazioni da miniature del genere, che farebbero in effetti pensare a una mediazione libraria, si trovano anche a L'Aquila, nel misterioso ciclo con storie di San Giorgio nella chiesa di San Pietro di Coppito (per i quali si veda S. PAONE, L'Aquila magnifica citade. Pittura gotica e tardogotica a L’Aquila e nel suo territorio, Roma, 2009, pp. 59-6o; EAD., Il Trecento angioino: la via degli Abruzzi e i rapporti con Napoli capitale, in S. PAONE, A. TOMEI, La pittura medievale nell'Abruzzo aquilano, Cinisello Balsamo, 2010, pp. 71-109: 96-102), soprattutto nella descrizione degli edifici, abitati da piccoli animali.

${ }_{33}$ Comunicazione scritta. Sul codice, si veda l'attento e particolareggiato studio di J. KUJAWIŃSKI, Alla ricerca del contesto del volgarizzamento della Historia Normannorum di Amato da Montecassino: il manoscritto francese 688 della Bibliothèque nationale de France, in Bullettino dell'Istituto Storico Italiano per il Medio Evo, 112, 2010, pp. 91-135. 

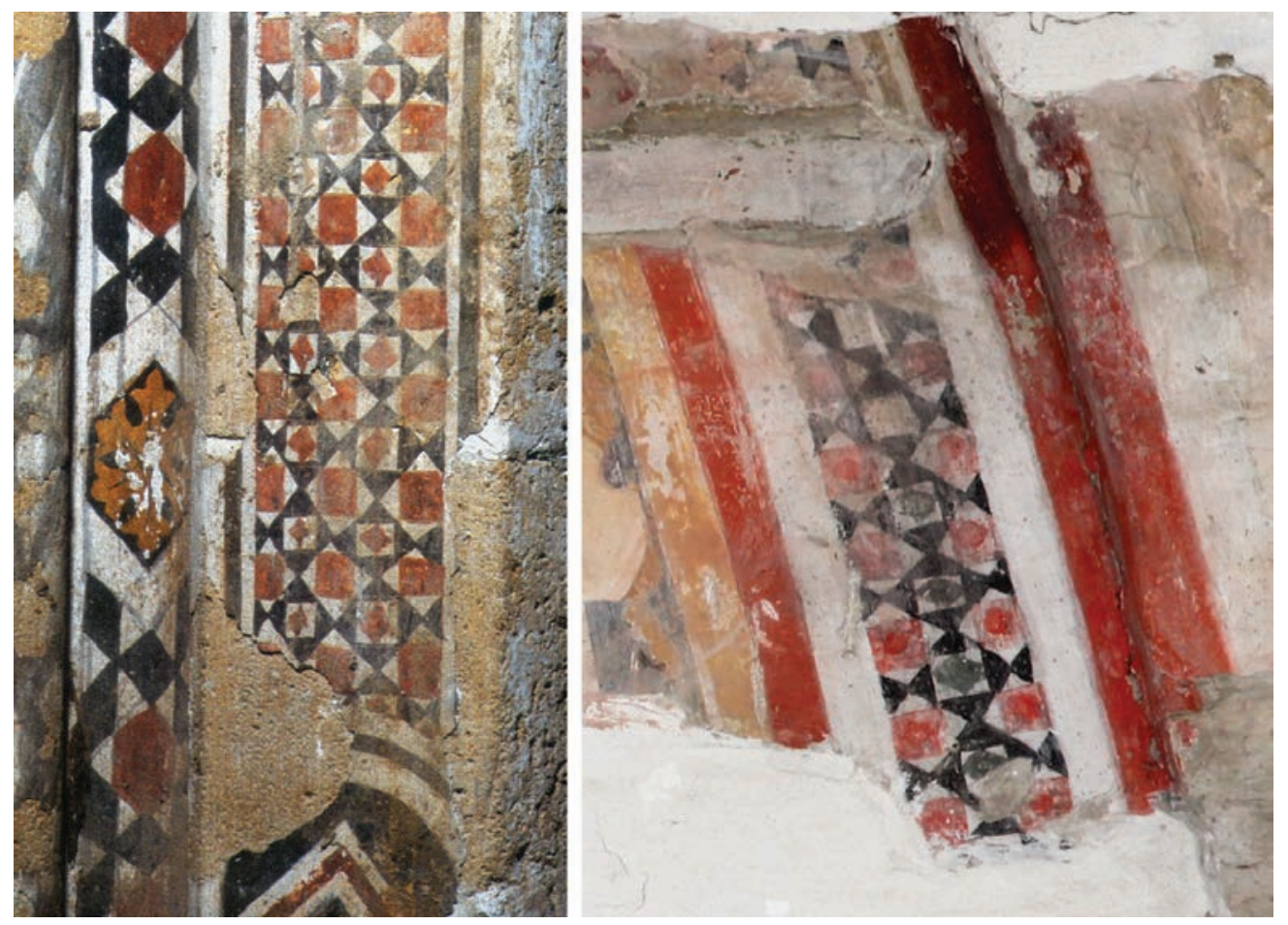

Fig. 22 - Napoli, Chiesa di Santa Chiara, fasce decorative (da P. LEONE DE CASTRIS, Giotto a Napoli); Penne, Chiesa di San Domenico, affreschi, fascia decorativa
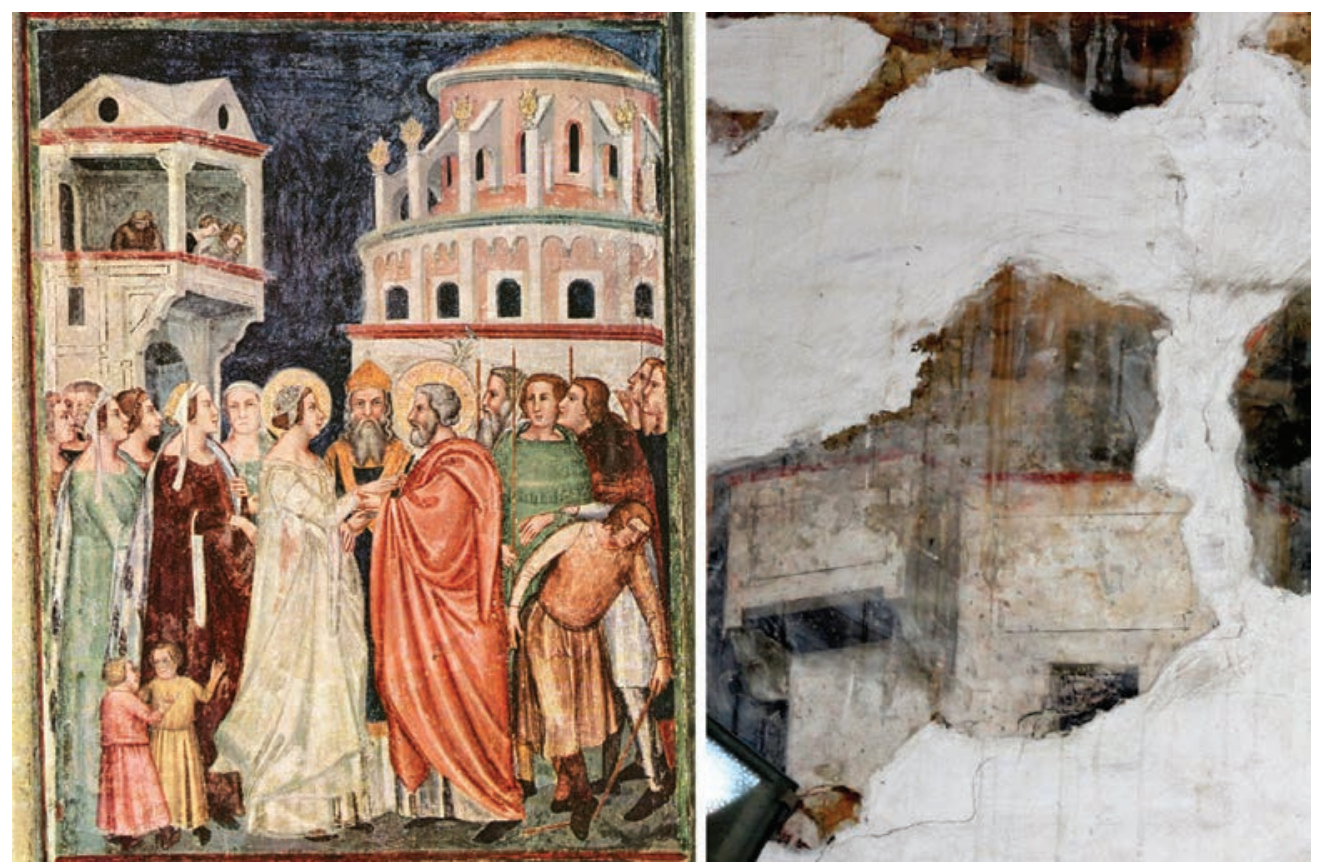

Fig. 23 - Napoli, Chiesa di San Lorenzo, Cappella Barrile, affreschi, Maestro di Giovanni Barrile, Sposalizio della Vergine (da F. BOLOGNA, I pittori alla corte angioina di Napoli); Penne, Chiesa di San Domenico, affreschi, Strage degli innocenti, dettaglio

fiorito e ridondante di gigli angioini: altro particolare che più tardi potrà rivelarsi di un certo interesse.

Il prototipo di tali decorazioni tassellate a pattern regolari può essere rintracciato a Napoli in ciò che resta degli affreschi giotteschi in Santa Chiara - ad esempio nei finestroni delle cappelle (fig. 22) ${ }^{34}$ e nella bordura superiore della Crocifissione nel vecchio convento ${ }^{35}$ - e persino (circostanza senz'altro significativa) nei dipinti cavalliniani che ornano il coro di Santa Maria Donnaregina ${ }^{36}$. I confronti, infatti, possono essere con molta facilità estesi alla pittura monumentale.

\footnotetext{
34 Si vedano le riproduzioni in P. LEONE DE CASTRIS, Giotto a Napoli, op. cit. (n. 22), pp. 122-123 figg. 69-72 e 74 .

35 Ibidem, p. 9 o fig. 43. La fuga di mensole intagliate è simile a quella che, a Penne, corre sul bordo superiore della teoria di santi nella chiesa degli Eremitani, e si discosta senz'altro, nella tipologia, da quelle riscontrabili a Offida e nei murali più direttamente collegabili a quel linguaggio, costituite assai spesso, per usare le parole di Leone de Castris, «da semplici e vistosi parallelepipedi sfaccettati» [P. LEONE DE CASTRIS, Gli affreschi del presunto Luca d’Atri. Chiesa di San Domenico, op. cit. (n. 16), p. 430].

${ }^{36}$ S. PAONE, Gli affreschi di Santa Maria Donnaregina Vecchia: percorsi stilistici nella Napoli angioina, in Arte Medievale, 3, 2004, 1, pp. 87-118 (si vedano le figg. 26 e 40, rispettivamente alle pp. 10o e 106). Altre riproduzioni sono ad esempio in F. BOLOGNA, I pittori alla corte angioina di Napoli, Roma, 1969, in part. tav. III-54 fig. 69. Interessanti sono anche le bordure nella cappella Brancaccio in San Domenico (ivi, tav. III-2, fig. 1). Su questi cicli si veda ora P. LEONE DE CASTRIS, Pietro Cavallini. Napoli prima di Giotto, Napoli, 2013.
} 

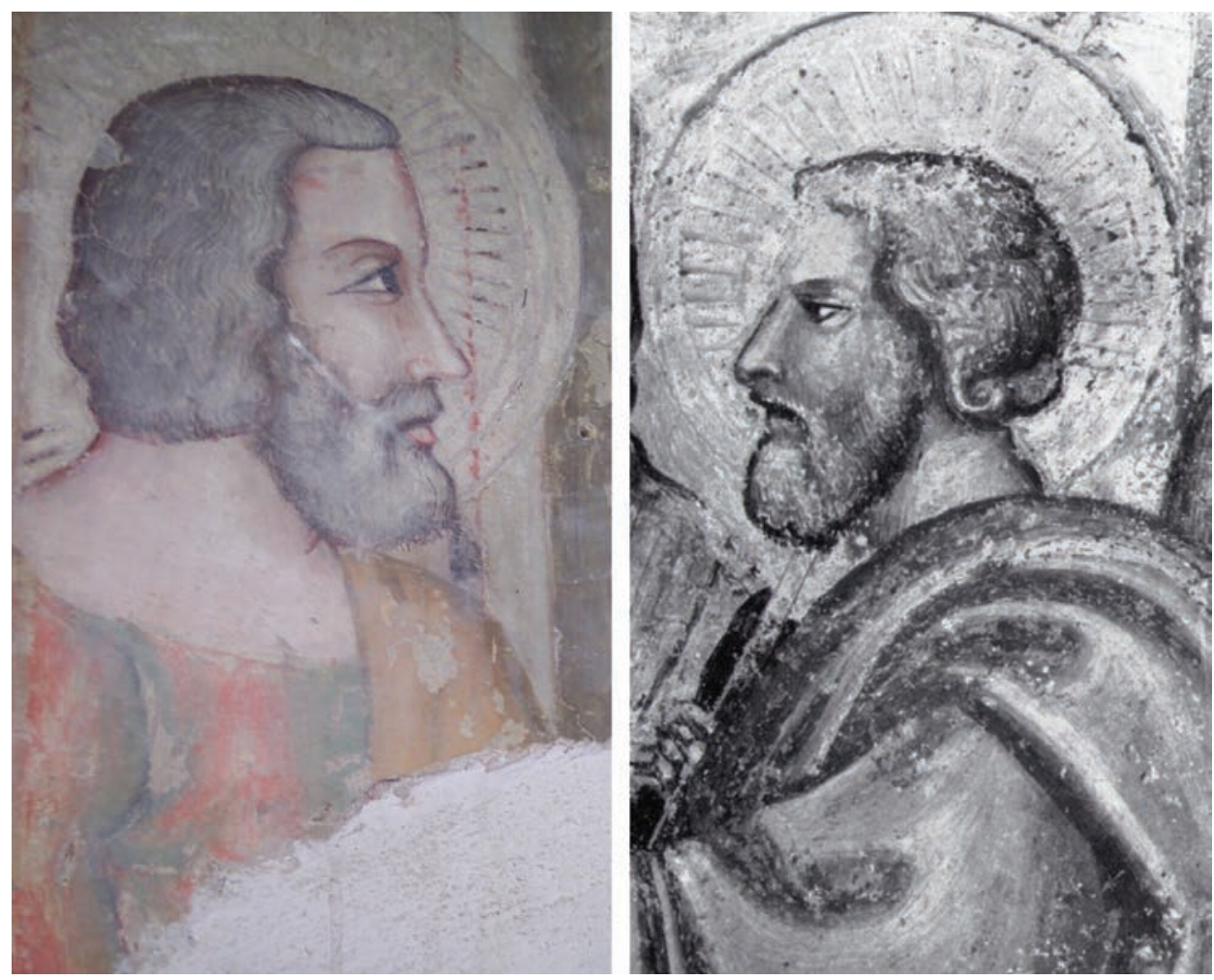

Fig. 24 - Napoli, Chiesa di San Lorenzo, Cappella Barrile, affreschi, Maestro di Giovanni Barrile, Sposalizio della Vergine, dettaglio (da F. BOLOGNA, I pittori alla corte angioina di Napoli); Penne, Chiesa di San Domenico, affreschi, Presentazione al Tempio, dettaglio
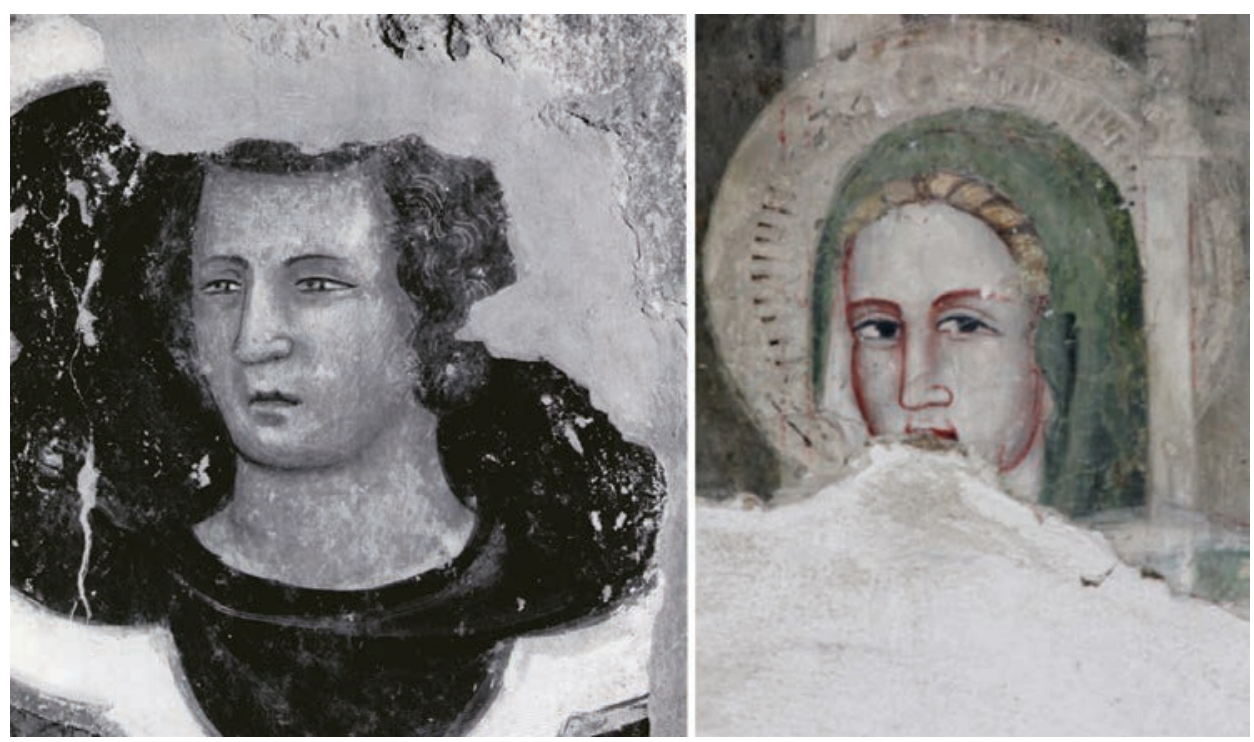

Fig. 25 - Napoli, Castel Nuovo, Cappella Palatina, affreschi, dettaglio (da F. BOLOGNA, I pittori alla corte angioina di Napoli); Penne, Chiesa di San Domenico, affreschi, Presentazione al Tempio, dettaglio dei contorni, sono caratteri che, come abbiamo visto, non si riscontrano nella sintetica, corsiva e allo stesso tempo raffinata visione degli affreschi di Offida e di quelli che altrove possono essere con maggior certezza collegati al linguaggio lì esperito (per i quali è senz'altro condivisibile il riferimento a una koiné "adriatica" o "umbro-marchigiana”, a un giottismo parallelo le cui radici sono da cercare appunto nell'Italia centrale), ma che, invece, dialogano in maniera diretta con lo svolgimento della pittura partenopea "post-giottesca" come esemplificata, ora che dell'attività del caposcuola fiorentino non resta più nulla, dal cosiddetto Maestro di Giovanni Barrile ${ }^{38}$ : molto simili appaiono infatti la concezione dello spazio (fig. 23) o i netti profili delle figure, con quelle calotte di capelli regolarissime, le barbe filamentose e la peculiare pastosità della materia pittorica (fig. 24), certo diversa da quella offidana, le cui tinte più tenui e quasi dilavate abbiamo già avuto modo di osservare. Restano senz'altro suggestive alcune consonanze, prima fra tutte l'aria di famiglia che apparenta i volti dagli occhi allungati e dal naso visto come di tre quarti, ma anche in questa occasione il modello può essere rinvenuto a Napoli, ad esempio in una delle teste superstiti nelle fasce decorative della Cappella Palatina di Castel Nuovo, già evocata e nella quale torneremo, attribuita non a caso proprio al Maestro di Giovanni Barrile (fig. 25) ${ }^{39}$. Non è neppure da escludere che saranno tali modi a influire sulla successiva pittura "picena", aggiungendo un elemento in più alla sintesi originale ${ }^{40}$. La difficile collocazione cronologica di quasi tutte le opere rende però arduo - e non immune da una certa perigliosa soggettività - argomentare questa ipotesi apparentemente così semplice.

Il ciclo di San Domenico si rivela, in definitiva, un ottimo esempio non soltanto della trasmissione di modelli

La tecnica stessa, l'impianto prospettico delle scene, ottenuto tramite linee incise e fili ribattuti ${ }^{37}$, nonché la durezza figurativi all'interno del Regno, ma anche, forse, della mobilità di artisti dalla capitale verso la sponda adriatica: non

\footnotetext{
37 Ben visibili ad esempio negli edifici della Strage degli Innocenti.

${ }^{38}$ F. BOLOGNA, I pittori alla corte angioina, op. cit. (n. 36), pp. 200-213; P. LEONE DE CASTRIS, Giotto a Napoli, op. cit. (n. 22), pp. 108, 113 nota 36 (con bibliografia precedente); M. DE GIORGI, Una Dormitio Virginis angioina poco nota ad Aversa: il caso di San Francesco delle Monache, in F. Abbate (ed.), Ottant'anni di un Maestro. Omaggio a Ferdinando Bologna, Pozzuoli, 2006, pp. 97-106: 99-102; S. PAONE, Giotto a Napoli. Un percorso indiziario tra fonti, collaboratori e seguaci, in A. Tomei (ed.), Giotto e il Trecento. I saggi, catalogo della mostra (Roma, 2009), pp. 179-195: 187-188.

39 Sulla decorazione della Cappella si veda naturalmente, con bibliografia precedente, P. LEONE DE CASTRIS, Giotto a Napoli, op. cit. (n. 22), pp. 168-197. ${ }^{40}$ In via del tutto ipotetica, si potrebbe pensare a un influsso degli affreschi pennesi su alcuni dettagli di quelli della chiesa domenicana di Teramo [per i quali si veda, naturalmente, P. LEONE DE CASTRIS, Gli affreschi del presunto Luca d'Atri. Chiesa di San Domenico, op. cit. (n. 16), che però si esprime, in part. a p. 431, a favore di una loro anteriorità rispetto al ciclo di cui ci stiamo occupando], ma la già notata distanza stilistica e la frammentarietà di entrambe le imprese rendono arduo argomentare tale ipotesi. In San Domenico a Penne è perduta la Crocifissione, ma quella teramana anticipa iconograficamente l'analoga pennese in Sant'Agostino (soprattutto nella presenza, tutt'altro che scontata, di Giuda impiccato ad un albero), tanto da farci supporre l'esistenza di un prototipo comune.
} 
è forse azzardato supporre che all'impresa abbiano partecipato, soprattutto nell'ideazione generale, artefici napoletani o comunque formatisi nella città partenopea al fianco dei maestri "post-giotteschi", i cui stilemi e prototipi dimostrano di conoscere assai bene. Ė a tal proposito significativa l'evocazione, da parte di Leone de Castris, del nome di Luca d'Atri, sconosciuto pittore il cui ricordo evanescente ci è tramandato dal celebre conterraneo Luca da Penne, giurista attivo a Napoli presso la corte di Giovanna ${ }^{41}$. In fondo, ad attraversare gli Appennini, e a tracciare una ideale "rotta" angioina, asse privilegiata per il traffico di materiali, persone e idee, erano gli stessi domenicani: tra questi, il pennese Nicola Titii Orfanellis, documentato «in conventu Neapolitano» nel 1363 e nel 1378 come Maestro degli studenti e poi come Lettore biblico ${ }^{42}$. E neppure è improbabile, in definitiva, che al cantiere si siano aggregati pittori formatisi in area picena, innescando una composita - e per nulla singolare, considerando il periodo storico - rete di scambi tra botteghe diverse: le vaghe similitudini già in parte sottolineate forniscono senz'altro indizi utili a supporre una fitta circolazione di artefici e stilemi, ma per il momento crediamo sia più prudente svincolare gli affreschi pennesi dalla comoda etichetta "Maestro di Offida" e considerarli appunto come il prodotto di una cultura partenopea, aggiornata su modelli databili all'incirca al quinto decennio del secolo, debitori a loro volta dei noti archetipi cavalliniani e giotteschi (e non immuni da tangenze di stampo più marcatamente goticheggiante). Del resto, a Penne, città che nel corso dei secoli non ha mai disdegnato di prodursi in volenterose emulazioni di ciò che si vedeva nella capitale, artisti e opere napoletani erano assai bene accolti, dal Quattrocento - almeno - fino al Settecento: basti pensare allo scultore Matteo $\mathrm{Capro}^{43} \mathrm{e}$ all'architetto Francesco di Sio ${ }^{44}$, presenze significative, e senz'altro influenti, che si pongono, con la tangibilità delle figure storiche reali, ai due estremi cronologici.
4. Una datazione dei nostri affreschi a metà del secolo potrebbe essere sostenuta non solo attraverso l'analisi formale ma anche per via storico-archivistica, seppure con tutte le cautele necessarie. Tra le carte relative alla soppressione murattiana, conservate nell'Archivio di Stato di Teramo, spicca un «notamento de' Libri Diplomi e Pergamene le più antiche che fra le altre si son rinvenute negli Archivi e Biblioteche de' soppressi conventi di Penne» ${ }^{45}$, che testimonia della gelosa custodia, fino alle soglie dell'età contemporanea, di un discreto numero di documenti medievali oramai perduti: in San Domenico fu inventariato, e segnato con il numero 1, un atto del 1350 in cui si annunciavano «i beni lasciati al convento per la costruzione del Coro» ${ }^{46}$. Originariamente allegata alla pratica, la pergamena risulta oggi irreperibile, e non è dunque prudente fantasticare troppo sul suo contenuto. È senz'altro suggestivo pensare che proprio in quegli anni la zona presbiteriale fu edificata, ricostruita o, appunto, decorata, ma per formulare ipotesi in tal senso dovremmo innanzitutto disporre di informazioni più precise sulla cronologia della chiesa, le cui prime fasi edilizie purtroppo ignoriamo ${ }^{47} ; \mathrm{e}$, cosa più importante, capire se il termine coro sia stato desunto dall'originale, e che quindi non si riferisca necessariamente all'abside, bensì, come nell'uso dell'epoca, alla zona del presbiterio riservata ai religiosi ${ }^{48}$, spesso situata di fronte all'altare maggiore ${ }^{49}$. La questione, dunque, è ancora aperta ${ }^{50}$.

Nonostante le notizie superstiti siano pochissime, sappiamo che la seconda metà del Trecento fu per i domenicani di Penne un periodo di particolare fervore: tra il 1352 e il 1360 un esponente fiorentino dell'ordine, Marco Ardinghelli, è vescovo della diocesi $i^{51}$; nel 1362 i frati prestano servizio nella importante e antica chiesa cittadina di San Comizio ${ }^{52}$ (e da qui proviene, tra l'altro, una tavola attribuita al Maestro di Offida)53; nel 1378 i confratelli della Compagnia di San Biagio (e si ricordi a proposito la già ipotizzata presenza di congregazioni laiche) sono ammessi alla partecipazione dei benefici dell'ordine ${ }^{54}$; nel 1391 un altro domenicano, Pietro

${ }^{41}$ E. CONTE, ad vocem Luca da Penne, in Dizionario Biografico degli Italiani, LXVI, Roma, 2007, pp. 251-254. Sulla menzione del giurista, si veda P. LEONE DE CASTRIS, Gli affreschi del presunto Luca d'Atri. Chiesa di San Domenico, op. cit. (n. 16), p. 450 nota 20, con bibliografia di riferimento.

${ }^{42}$ B. CARDERI, I Domenicani nella diocesi di Penne, op. cit. (n. 3), pp. 672 e 739-740.

${ }^{43}$ F. ACETO, Matteo Capro "de Neapoli" a Penne, in Dalla valle del Fino alla valle del medio e alto Pescara, op. cit. (n. 1), pp. 436-438; V. FRATICELLI, Matteo Capro da Napoli, in Studi Medievali e Moderni, 18, 2014, 1-2, pp. 223-256.

${ }^{44}$ F. BATTISTELLA, Note su alcune fabbriche attribuite a Francesco di Sio, op. cit. (n. 1).

${ }^{45}$ Archivio di Stato di Teramo, Intendenza francese, Secondo ufficio, Sez. I, busta 196, fascicolo 4652, 1809-1810, doc. 4. B. CARDERI, I Domenicani nella diocesi di Penne, op. cit. (n. 3), pp. 744-746.

${ }^{46}$ Ibidem, pp. 739, 745. Così nell'originale:«Pergamena del 1350, nella q(ua)le per stromento si annunciano i beni lasciati al Convento per la costruz(ione) del Coro. Segnata col num(ero) 1». La pergamena è purtroppo perduta. Ringrazio le dottoresse Luciana D'Annunzio e Carmela Di Giovannantonio dell'Archivio di Stato di Teramo per la consueta, piacevole collaborazione.

${ }^{47}$ Come già visto in apertura, il convento era, già nel penultimo decennio del XIII secolo, «completo (formale), funzionante con i suoi tre officiali ordinari, cioè Priore, Professore (Lettore) ed Economo (Sindaco); la data ha la sua importanza perché gli atti menzionano con Penne altri due conventi della regione, uno a L'Aquila e l'altro a Sulmona, tutti e tre soggetti all'annuale ispezione, che nel 1283 sarà fatta da P. Paolo d'Aversa» [B. CARDERI, I Domenicani nella diocesi di Penne, op. cit. (n. 3), pp. 670-671]. Si accenna al convento: non sappiamo, però, quando la chiesa attuale fu edificata.

${ }^{48}$ Ringrazio la professoressa Giovanna Valenzano per aver attirato l'attenzione su questo punto.

${ }^{49}$ Sull'argomento si veda M.C. ROSSI, L'architettura medievale della Collegiata dei santi Cesidio e Rufino attraverso i documenti e le fonti, in G. Curzi (ed.), La collegiata dei santi Rufino e Cesidio a Trasacco: un santuario nella Marsica, Roma, 2015, pp. 19-30: 23, con riferimento alla definizione registrata in C. DU CANGE et alii, Glossarium mediae et infimae latinitatis, éd. augm., Niort, 1883-1887, II, col. 316a: «Pars Ecclesiæ in qua Clerus consistit, ac concinit». Il Du Cange attesta anche il significato di capitulum (ivi, col. 316b).

${ }^{50}$ A questo documento, Martina Papilli ha convincentemente collegato il gruppo di corali provenienti dalla chiesa (tesi di laurea discussa presso l'Università di Chieti nell'Anno Accademico 2002-2003, relatrice la professoressa Francesca Manzari) e oggi custoditi presso l'Archivio Storico Diocesano. C. TEDESCHI, Una biblioteca nascosta. Frammenti di una bibbia atlantica e di altri codici nell'Archivio diocesano di Penne e Pescara, in C. Tristano (ed.), Frammenti di un discorso storico, in corso di stampa.

${ }^{51}$ B. CARDERI, I Domenicani nella diocesi di Penne, op. cit. (n. 3), pp. 672, 739.

${ }^{52}$ Ibidem.

${ }^{3}$ Conservata nel Museo Nazionale dell'Aquila. F. BOLOGNA, Altre aggiunte al Maestro di Offida, in Dalla valle del Piomba alla valle del basso Pescara, Pescara, 2001 (Documenti dell'Abruzzo Teramano, V/1), pp. 297-298.

54 B. CARDERI, I Domenicani nella diocesi di Penne, op. cit. (n. 3), pp. 672-673, 740. 
Scaglia di Roma, siede allo scanno episcopale55; quattro anni dopo, il pennese Petrucius è investito presule di Scala, sede suffraganea di Amalfi ${ }^{5}$. Pur considerando i lunghi, inevitabili silenzi documentari, è dunque possibile rintracciare le maglie di una rete assai fitta di contatti e scambi, a testimonianza di un contesto vivace, animato dall'attività di confraternite e vibrante di esperienze sociali.

Prima di uscire dalla chiesa, e congedarci dagli affreschis7, vorremmo però discutere per un istante un ultimo quesito iconologico, utile forse a tracciare una ulteriore pista di lettura: com'è ben noto, un grande ciclo comprendente storie bibliche e apocalittiche si trovava in Santa Chiara a Napoli - monastero doppio legato alla liberalità di Sancia di Maiorca $^{58}$, la cui immagine di donatrice e devota spicca ancora oggi in un affresco della sala capitolare ${ }^{59}$ - e altri soggetti dell'Antico e del Nuovo Testamento erano ben visibili nella Cappella Palatina di Castel Nuovo ${ }^{60}$, della quale abbiamo già notata la vaga ma suggestiva consonanza icnografica con l'antica aula domenicana.

Ebbene, se riandiamo con la mente alla rendita concessa nel 1294 dalla corona, e al prestigioso nesso storico con Carlo II, il cui ricordo era ancora ben vivo in epoca moderna, viene da chiedersi se nel ciclo non fosse palese una esplicita connotazione filoangioina, tesa non solo a emulare e in qualche modo citare le committenze regie ma, soprattutto, a ribadire anche visivamente tale legame ideologico. In una testimonianza del 31 marzo $1650^{61}$, precedente al rinnovamento barocco, il priore Valeriano Barzanti da Lucca, e i padri Reginaldo Puccini, Egidio Giovannini e Vincenzo Ponzi (lucchesi anch'essi, e rispettivamente Lettori e Sindaco) ci avvertono che «per l'antichità, non habbiamo cosa certa» riguardo la fondazione del cenobio, ma, ricollegandosi alla tradizione orale che già conosciamo, aggiungono una frase per noi straordinariamente evocativa: «si crede però sia uno delli conventi fatti edificare da Carlo Secondo Re di Napoli per li molti gigli che in diverse parti del convento si trovano scolpiti e dipinti ${ }^{62}$. Purtroppo, a parte il vago accenno ad una epigrafe recante la data del 1330, relativa alla costruzione della cisterna ${ }^{63}$, i padri secenteschi non ci forniscono indizi utili a ricostruire l'aspetto di questi ultimi, crepuscolari frammenti della fase medievale dell'edificio, definitivamente obliterati nel secolo successivo. Del resto, gli stessi frati assicurano che, nel corso degli anni, «detto convento si è andato sempre avanzando (in riguardo al luogo) in fabrica et ornamento di chiesa maravigliosamente ${ }^{64}$.

Se la presenza ridondante dei gigli, che richiama alcune miniature più su discusse, è oramai un ricordo scritto, i pochi frammenti superstiti ci parlano ancora oggi di un legame con gli angioini e con il pittore che si accinse ad istoriare con scene della Bibbia e dell'Apocalisse gli edifici simbolo della capitale: Giotto. L'angelo annunciante che spiegava le sue ali multicolori (fig. 16) si direbbe quasi tramandare l'ombra di una estrema inventio dell'artista fiorentino che, abbandonando per un istante il riscoperto spazio terreno, e osservando il grande tabellone apocalittico cavalliniano in Santa Maria Donnaregina, diede probabilmente vita a enormi, sovrannaturali visioni mistiche, la cui eco è forse nelle tavole di Stoccarda. Visioni che, non a caso, suggerirono a Ghiberti l'idea di una diretta consulenza dantesca ${ }^{65}$. Le corrispondenze puntuali con le miniature napoletane, dagli schemi iconografici al peculiare repertorio decorativo, lasciano pensare, in definitiva, che gli affreschi citassero in maniera consapevole e programmatica gli scomparsi prototipi giotteschi. Il ciclo di Penne costituirebbe dunque, da questo punto di vista, la pallida e ancora una volta frammentaria testimonianza dell'opera del "sovrano maestro" a Napoli e della sua fortuna.

\footnotetext{
55 Ibidem, pp. 672, 740-741.

${ }^{56}$ Ibidem, pp. 672, 741.

${ }^{57}$ Della maniera inaugurata in San Domenico abbiamo a Penne l'indizio di una notevole evoluzione: ci riferiamo agli affreschi ancora oggi visibili nel coro della chiesa di Sant'Agostino (P. DI SIMONE, Gli affreschi della chiesa di Sant'Agostino a Penne, in Studi Medievali e Moderni, 13, 2009, 25, pp. 73-104, con bibliografia precedente), con una teoria di santi assai vicina nei modi al ciclo domenicano, che nella seconda metà del secolo si disponeva attorno alla monofora centrale, e una grande Crocifissione già al corrente dei modi degli affreschi dell'Incoronata e con qualche intrigante anticipazione del Maestro di Loreto Aprutino (di cui mancano però quel forte espressionismo che porta a conseguenze estreme gli stilemi bolognesi, qui apparentemente assenti, nonché gli esotismi di marca "internazionale") e di miniatori come quello del Messale Acquaviva di Atri (si veda il profilo muliebre nel bas-de-page di f. 284r, da confrontare con l'astante all'estrema sinistra nell'affresco. Sul Messale Acquaviva: C. PASQUALETTI, scheda n. 44, in G. Curzi, F. Manzari, F. Tentarelli, A. Tomei (ed.), Illuminare l'Abruzzo. Codici miniati tra Medioevo e Rinascimento, Pescara, 2012, pp. 248-249). Si tratta infatti di un dipinto probabilmente realizzato alla fine del XIV secolo - come suggerisce anche la foggia delle armature dei soldati che si accalcano sul Calvario - che segue cronologicamente la teoria di santi cui in parte si sovrappone (sono da abbandonare definitivamente le perplessità avanzate nel saggio del 2009, citato in apertura di nota, ferme restando le osservazioni stilistiche sulle similitudini tra i due strati, la cui cultura figurativa di base è la stessa), come ben rilevato da Ferdinando Bologna [per una sintesi delle opinioni dello studioso sull'affresco: F. BOLOGNA, Crocifissione di Antonio da Atri e altri affreschi. Chiesa di Sant'Agostino. Penne, in Dalla valle del Fino alla valle del medio e alto Pescara, op. cit. (n. 1), pp. 485-489, in part. p. 486].

${ }_{58}^{8} \mathrm{Si}$ veda ancora una volta il fondamentale P. LEONE DE CASTRIS, Giotto a Napoli, op. cit. (n. 22), pp. 65-167.

59 V. LUCHERINI, Regalità e iconografia francescana nel complesso conventuale di Santa Chiara a Napoli: il Cristo in trono nella sala capitolare, in Ikon, 3, 2010, pp. 151-168.

${ }^{60}$ P. LEONE DE CASTRIS, Giotto a Napoli, op. cit. (n. 22), pp. 168-216.

${ }^{61}$ Il documento, custodito nell'Archivio Segreto Vaticano (Congregazione sopra lo stato Regolare. Domenicani 1650, tomo I, I Tavola della Provincia dell'Abruzzo, 7, pp. 15-18), è trascritto in B. CARDERI, I Domenicani nella diocesi di Penne, op. cit. (n. 3), pp. 819-824. Ci riferiamo a tale edizione, e non all'originale.

${ }^{62}$ Ibidem, p. 819. I corsivi sono nostri.

${ }^{63}$ Ibidem, p. 820.

${ }^{64}$ Ibidem.

${ }^{65}$ P. LEONE DE CASTRIS, Giotto a Napoli, op. cit. (n. 22), p. 65.
} 